\title{
Artificial neural network with Taguchi method for robust classification model to improve classification accuracy of breast cancer
}

\author{
Md Akizur Rahman ${ }^{\text {Corresp., }}{ }^{,}$, Ravie chandren Muniyandi ${ }^{1}$, Dheeb Albashish ${ }^{2}$, Md. Mokhlesur Rahman ${ }^{1}$, Opeyemi \\ Lateef Usman $^{1}$ \\ ${ }^{1}$ Research Center for Cyber Security, Faculty of Information Science and Technology, Universiti Kebangsaan Malaysia, 43600 Bangi, Selangor, Malaysia \\ 2 Computer Science Department, Prince Abdullah Bin Ghazi Faculty of Information and Communication Technology,, Al-Balqa Applied University,, As-Salt, \\ Salt, Jordan \\ Corresponding Author: Md Akizur Rahman \\ Email address: akiz.akizur@gmail.com
}

Artificial neural networks (ANN) perform well in real-world classification problems. In this paper, a robust classification model using ANN was constructed to enhance the accuracy of breast cancer classification. Taguchi method was used to determine the suitable number of neurons in a single hidden layer of the ANN. The selection of a suitable number of neurons helps to solve the overfitting problem by affecting the classification performance of an ANN. With this, a robust classification model was then built for breast cancer classification. Based on the Taguchi method results, suitable number of neurons selected for hidden layer in this study is 15 , which was used for the training of the proposed ANN model. The developed model was benchmarked upon the Wisconsin Diagnosis Breast Cancer Dataset, popularly known as the UCl dataset. Finally, the proposed model was compared with seven other existing classification models, and it was confirmed that the model in this study had the best accuracy at breast cancer classification, at $98.8 \%$. This confirmed that the proposed model significantly improved performance. 


\section{Artificial Neural Network with Taguchi Method for 2 Robust Classification Model to Improve Classification 3 Accuracy of Breast Cancer}

4

5

6

7

8

9

10

11

Md Akizur Rahman ${ }^{1 *}$, Ravie Chandren Muniyandi ${ }^{1}$, Dheeb Albashish ${ }^{2}$, Md. Mokhlesur Rahman $^{1}$, and Opeyemi Lateef Usman ${ }^{1}$

${ }^{1}$ Research Center for Cyber Security, Faculty of Information Science and Technology, Universiti Kebangsaan Malaysia, 43600 Bangi, Selangor, Malaysia

2 Computer Science Department, Prince Abdullah Bin Ghazi Faculty of Information and Communication Technology, Al-Balqa Applied University, Salt, Jordan

Corresponding Author:

Md Akizur Rahman ${ }^{*}$

Research Center for Cyber Security, Faculty of Information Science and Technology, Universiti Kebangsaan Malaysia, 43600 Bangi, Selangor, Malaysia

Email address: akiz.akizur@gmail.com

\section{Abstract}

Artificial neural networks (ANN) perform well in real-world classification problems. In this paper, a robust classification model using ANN was constructed to enhance the accuracy of breast cancer classification. The Taguchi method was used to determine the suitable number of neurons in a single hidden layer of the ANN. The selection of a suitable number of neurons helps to solve the overfitting problem by affecting the classification performance of an ANN. With this, a robust classification model was then built for breast cancer classification. Based on the Taguchi method results, suitable number of neurons selected for hidden layer in this study is 15 , which was used for the training of the proposed ANN model. The developed model was benchmarked upon the Wisconsin Diagnosis Breast Cancer Dataset, popularly known as the UCI dataset. Finally, the proposed model was compared to seven other existing classification models, and it was confirmed that the model in this study had the best accuracy at breast cancer classification, at $98.8 \%$. This confirmed that the proposed model significantly improved performance.

\section{Introduction}

Despite current technological advances, medical sciences remain limited in their ability to contain and treat cancer diseases. The containment and treatment of cancer diseases form the crux of the medical science community's efforts at technological advancements. Cancer is known to be the 
39

40

41

42

43

44

45

46

47

48

49

50

51

52

53

54

55

56

57

58

59

60

61

62

63

64

65

66

67

68

69

70

71

72

73

74

75

76

77

78

most severe complex of diseases when it comes to mortality rates, and breast cancer is the most common leading cause of cancer death in women. Many women above 40 years old suffer from breast cancer. It is prevalent to the point that it has been identified as the second most deadly unavoidable disease for women in this age bracket ( $>40$ years old) [1].

In order to properly treat breast cancer, its identification and diagnosis during the early stages are crucial. The traditional approach to diagnosis is highly reliant upon the experience of the attending physician(s). The reliability of physicians' experience and visual inspections is questionable due to the high probability of human error. There is also an extremely large volume of datasets (Big data) with poor quality and redundant information, making the diagnosis of cancer at an acceptable level of accuracy a complex affair despite physicians' vast experience. In an attempt to improve the accuracy of cancer classification, a computer-aided diagnostic (CAD) system has been used to assist physicians [2][3][4][5][6][7][8][9][10]. The use of the CAD system for classification is improving the medical diagnosis process. Classification systems can help not only to minimize possible mistakes associated with a lack of experience among physicians, but also to provide accurate information for the examination(s) of medical datasets [2][11].

Artificial neural network (ANN) models, which are inspired by the complex, interconnected neural structure of the brain, have been proposed for classification tasks. In ANN, learning is realized via experience, and behavior modifications are in response to environmental stimuli. The model also generalizes from previous examples to address new problems. Figure 1 shows the three layers of an ANN: input, hidden, and output. Two layers are in communication with the external environment. The input layer receives input signals directly from the environment, which are then processed by the network, while the output layer of the network delivers the processed results to the (outward) environment. The number of neurons in the output layer is directly linked to a particular number of tasks the neural network was designed to carry out. The intermediate layer linking the input and output layers is called the hidden layer. This layer contains a function known as the activation or transfer function, which performs nonlinear activation on the sum of the weighted inputs from the preceding layer. It is associated with the hidden neurons, which are neurons that are absent from both the input and output layers [12]. Hidden neurons result in two outcomes: overfitting and underfitting. The large number of hidden neurons can be a potential cause of the latter, while overfitting occurs when there are multiple unnecessary neurons present in the hidden layer [12]. Underfitting occurs when the number of hidden neurons is lower than what is required to model a problem dataset. The small number of neurons in the hidden layers are pressured to properly detect highly complex signals [12]. A neural network architecture is dictated by the number of its hidden layers, due to the fact that it is directly linked to the external environment [13]. The determination of the accurate number of hidden layers is crucial for increasing the accuracy of cancer classification. One method that is effective for the determination of the suitable number of hidden layers is the Taguchi method [14].

The Taguchi method [14] has gained prominence in several research works due to its focus on optimization problems. It performs well despite uncertain conditions, producing low-cost outputs and robust parameter design via the integration of traditional engineering with statistics 
79

80

81

82

83

84

85

86

87

88

89

90

91

92

93

94

95

96

97

98

99

100

101

102

103

104

105

106

107

108

109

110

111

112

113

114

115

116

117

118

for approximation and performance enhancement in multiple cases. For processes and parameter arrangement, the Taguchi method utilizes the statistical investigational architecture. In a robust experimental setup and design, processes or products can be investigated by altering design-related factors. This experimental design helps to proficiently and consistently analyze outputs [14][15].

Therefore, this study proposes the Taguchi method for the parameter optimization for an ANN algorithm, specifically, for the determination of the optimal number of neurons in a single hidden layer. This helps to increase the accuracy of cancer classification.

The remainder of this paper is organized as follows: Section 2 introduces related works, while Section 3 details the experimental dataset, the Taguchi method, and ANN. Section 4 discusses the proposed algorithm for breast cancer classification. Section 5 presents the experimental results and discussion. Finally, Section 6 concludes the work and suggests future research possibilities.

\section{Related Studies}

The most important requirements for machine learning techniques in medical diagnosis and cancer classification are accuracy and reliability. This section presents related studies that were previously conducted in this field, emphasizing ANN performance improvement and cancerous dataset classifications. The literature offers many examples of research works employing an experimental design to determine the suitable number of parameters, which could influence the performance of the ANN. To efficiently establish an ANN's parameters, Khaw et al. [16] proposed the Taguchi method using two sets of simulated datasets in order to increase the accuracy and convergence speed of the back-propagation network (BPN). Peterson et al. [17] utilized the Taguchi method to determine the causes of faults in the BPN, while Yang and Lee [18] minimized the ANN training duration using the Taguchi method. Packianather et al. [19] reported the outcome of parametric design on the performance of a neural network for a wood veneer examination using the Taguchi method. For the purpose of designing a multilayer feed-forward neural network, Yum and Kim [20] used Taguchi's active method to account for noise. Tortum et al. [21] utilized the Taguchi method to determine the suitable combination of effectual parameters, and reported the consequences of the performance criteria of every parameter in the neural network. Sukthomya and Tannock [22] utilized the Taguchi method to determine the suitable combination of effectual parameters in a neural network. Jung et al. [23] employed the Taguchi method to develop a dynamic parameter design that relies on an ANN. Becherer et al. [24] used the parametric finetuning technique with a convolutional neural network $(\mathrm{CNN})$ for the purpose of image classification.

On the topic of breast cancer diagnosis and classification, Zheng et al. [25] proposed a hybridization of the k-means algorithm and support vector machine (K-SVM) for breast cancer diagnosis. The results achieved 97.38\% accuracy using the Wisconsin Diagnostic Breast Cancer (WDBC) dataset. Orkcu et al. [26] compared the performance of a real-coded genetic algorithm, back-propagation neural network (BPNN), and binary coded genetic algorithm models using the breast cancer datasets, and reported accuracies of $96.50 \%, 93.10 \%$, and $94.00 \%$, respectively. Salama et al. [27] used the classifiers Naive Bayes (NB), sequential minimal optimization (SMO), 
119 decision tree (J48), multi-layer perception (MLP), and instance-based for k-nearest neighbor (IBK$120 \mathrm{NN}$ ) for breast cancer classifications. The experiment, which adopted a confusion matrix based on 121 the 10-fold cross-validation method, using datasets from three distinct databases, showed that the 122 highest classification accuracy of $97.70 \%$ was realized using the sequential minimal optimization 123 (SMO) model.

On the other hand, Malmir et al. [28] used an imperialist competitive algorithm (ICA) with multilayer perceptron (MLP) network and particle swarm optimization (PSO) for breast cancer classification. This study achieved $97.75 \%$ and $97.63 \%$ of classification accuracies for MLP and PSO, respectively. Koyuncu et al. [29] attempted to achieve higher classification accuracy using a breast cancer dataset and Rotation Forest artificial neural network (RF-ANN) classifier. The result from the analysis of the above classifier was $98.05 \%$ accurate. Aalaei et al. [5] compared the performance of a genetic algorithm with two similar classifiers, namely a particle swarm classifier and ANN, using the WDBC datasets without feature selection. The experiment reported accuracies of $96.40 \%, 96.50 \%$, and $96.10 \%$, respectively.

Osman et al. [30] proposed Ensemble Boosting Learning (EBL) method for breast cancer classification. The author used UCI different breast cancer dataset including Wisconsin Breast Cancer Diagnostic (WBCD) dataset. The proposed method able to achieve the classification accuracy of $97.0 \%$ using 10-fold cross-validation with Radial Based Function Neural Network (RBFNN) classifier. Xue et al. [31] proposed the PSO technique with novel initialization and updated mechanisms hybridized with a KNN classifier for breast cancer classification. Author performed on WDBC dataset by using for training 70\% and 30\% for testing with 10-fold crossvalidation and able to achieve the classification accuracy of 92.98\%. Nekkaa et al. [32] proposed a classification model by using Memetic algorithm (MA) with Support vector machine (SVM) to address the classification problem. The authors used particular datasets to compare certain popular classifiers including WDBC dataset. The model achieved $97.85 \%$ of classification accuracy.

Idri et al. [33] were developed heterogeneous ensembles-based classification technique for breast cancer classification. The heterogeneous ensembles included support vector machines (SVM), multilayer perceptron (MLP), and decision trees (DTs) to evaluate the classification performance. The author was built three groups of heterogeneous ensembles using three single classifiers optimized by GS, PSO, and UC Weka. From the technique, the author performed by using UCI breast cancer dataset including WDBC and achieved the highest classification accuracy of $98.07 \%$ by GSVM. Jijitha et al. [34] performed on six types of different breast cancer dataset including BCWD (Breast Cancer Wisconsin Diagnostic) dataset using machine learning techniques. For breast cancer classification the author used K-Nearest Neighbor (K-NN) and Logistic Regression (LR) technique. From BCWD dataset achieved a classification accuracy of $96.5 \%$ and $97.02 \%$ by LR and K-NN. Thiyagarajan et al. [35] investigated the application of machine learning methods for classification of breast cancer. For an investigation of breast cancer classification performance was used different machine learning methods which are included as dataset and the highest classification accuracy of 96.2\% from the ANN method. Quy et al. [36] 
159

160

161

162

163

164

165

166

167

168

169

170

171

172

173

174

175

176

177

178

179

180

181

182

183

184

185

186

187

188

189

190

191

$192 \quad i i$

193 iii

194 iv

$195 v$

196

197

198 viii

1

applied Machine Learning-Based Evolutionary Neural Network Approach for breast cancer classification. The author focused on parameter optimize of neural network (NN) model using Adaptive Particle Swarm Optimization (APSO) algorithm. For the NN used 20 neurons in a single hidden layer. The WDBC dataset was used and partitioned $70 \%$ for training and $30 \%$ for testing. The model was trained by Back-propagation (BP), classical PSO and APSO respectively. From the experimental result achieved the highest classification accuracy of $98.24 \%$ by APSO-NN.

From the literature, it can be concluded that ANN is utilized since it involves pattern recognition and data classification. The most important advantage of ANN with regard to the classification problem in multisource databases has been solved. ANN is an established tool in data classification that is easy to utilize and implement. In previous research, two limitations were identified: (1) low classification accuracy [27][30][31][32], and (2) neuron selection in the hidden layer [5][36]. This study proposes 15 neurons in single hidden layer of ANN which can assist to improve the accuracy of breast cancer classification.

\section{Materials \& Methods}

\section{Experimental Dataset}

This research used the Wisconsin Diagnosis Breast Cancer (WDBC) dataset from the UCI Machine Learning Repository atavailable http://archive.ics.uci.edu/ml/datasets/Breast+Cancer+Wisconsin+(Diagnostic)] to differentiate malignant tumors from normal tumor samples. The dataset was used to compare normal tumors with cancerous (malignant) tumors [37]. The dataset contains 32 features, namely ID, diagnosis, and 30 real-valued input features, followed by 569 samples, of which 357 are normal and 212 are cancerous, with zero missing attribute values. The dataset features were originally computed from a digitized image of a fine needle aspirate (FNA) of a breast mass by the first user and have become a reference dataset for many recent studies on breast cancer classification. The attribute information is as follows:

\section{Attribute Information:}

ID number

2 Diagnosis ( $M=$ Malignant, $N=$ Normal)

3 to 32 Ten real-valued features are computed for each cell nucleus:

Radius (mean of distances from center to points on the perimeter)

Texture (Standard deviation of gray-scale values)

Perimeter

iv $\quad$ Area

$v \quad$ Smoothness (Local variation in radius lengths)

vi Compactness (Perimeter^2 / area - 1.0)

vii Concavity (Severity of concave portions of the contour)

viii Concave points (Number of concave portions of the contour)

Peer] Comput. Sci. reviewing PDF | (CS-2020:08:52384:1:1:NEW 23 Nov 2020) 
199 ix Symmetry

$200 x \quad$ Fractal dimension ("Coastline approximation" - 1)

201 For this study, our experimental dataset was divided into training, validation, and testing datasets

202 using four different partitions based on the Taguchi method (Followed by Equation 1 and Table

203 1). The partitions were chosen based on the experimental performance. The dataset partitions are 204 as follows: The first partition contains 50, 25, and 25; the second partition contains 60, 20, and 20; 205 the third partition contains 70, 15, and 15; and, finally, the fourth partition contains 80, 10, and 10, 206 where the first number represents the training set, the second number represents the validation set, 207 and the third number represents the testing set for each partition, respectively. Section 3.2 presents 208 details of how the Taguchi method was used. In Section 4, we discuss the performance of the 209 different dataset partitions used (Table 2).

210

\section{Taguchi Method}

212 The Taguchi method is a robust experimental design [14] process that can be analyzed and 213 improved upon by altering the relative design factors. It is also called the statistical method and 214 can be used to realize the highest product quality. The Taguchi method utilizes a three-stage 215 method: system design, parameter design, and tolerance design. In the system design, suitable 216 working levels of design factors are accounted for. For design and testing, a system needs to be 217 based on the designers' judgement of factors such as materials, parts, nominal products, processes, 218 or parameters, based on the latest technology. The parameter design is used to determine 219 parametric levels in order to enhance the accuracy of the process being considered. Tolerance 220 design is used to fine-tune the results. As a commonly used robust design approach, the Taguchi 221 method has two mechanisms: Orthogonal array and signal-to-noise ratio (SNR) [38], for 222 improvement and analysis. To minimize experimental efforts, Orthogonal array is mainly utilized, 223 using $N$ number of design parameters.

An Orthogonal array is very useful, as it gives an extensive investigation of associations among all design factors and reasonably adjusts the methodical correlations of various dimensions of each factor. An orthogonal array is a two-dimensional array. Each column represents a certain design parameter, while each row denotes an experimental test with an actual arrangement of various levels for all of the design factors. In this research, the two-level Orthogonal array for determining optimal neurons is shown in Equation (1):

$$
L_{M}\left(2^{N}\right)
$$

where $N$ is the number of columns in the Orthogonal matrix. $M=2 K\left(M>N, K>\log _{2}(N) ; M\right.$ is the number of expected experimental trials, and $K$ is an integer. Base 2 is the number of levels of every design parameter. In this study, there are 20 numbers of columns in the Orthogonal matrix (where F1 to F20 indicate the design factor the number of neurons in the hidden layer), and a twolevel Orthogonal array was used for selecting appropriate number of neurons. The two-level Orthogonal array was created using $\mathrm{L}_{21}\left(2^{20}\right)$, as shown in Table 1.

236

Only 21 experimental trials are required for evaluation, analysis, and improvement. Conversely, all possible combinations of 20 design factors (i.e., $2^{20}=1,048,576$ ) should be 
238

239

240

241

242

243

244

245

246

247

248

249

250

251

252

253

254

255

256

257

258

259

260

261

262

263

264

265

266

267

268

269

270

271

272

273

274

275

276

277

accounted for in the full factorial experimental design, which is frequently inapplicable in practice [39] (for neuron selection: 1: selected, 0: not selected).

This research used an Orthogonal array mechanism to analyze and enhance the ANN algorithm performance by determining the optimal number of neurons in hidden layer. If a particular target has $N$ different design factors, $2^{N}$ possible experimental trials will be considered in the full factorial experimental design.

Table 1. Orthogonal array (OA).

\section{Artificial Neural Network:}

An artificial neural network (ANN) [11] is a machine learning approach, which models the human brain with a number of artificial neurons and interconnected associations. The neurons in ANNs tend to have fewer connections relative to a biological neural system. Figure 1 illustrates the basics of the ANN architecture.

Figure 1. The basic architecture of an ANN.

Neurons are highly interconnected computational units inspired by the mammalian brain. The ANN system consists of the smallest processing nodes called neurons, or processing elements. To obtain outputs, a function called Sigmoid activation works on inputs and connects the weights with the help of neurons. The connections between weight (w) values and single nodes are called biases (b). The iterative flow of training data determines weight values throughout the network. During the training phase, weight values are verified until the network is able to detect a particular cluster using criteria for typical input data. Also, these weights assign the link relating one layer of neurons to another. Hence, changes in the relationship between input and output take place repeatedly, along with changes in weight values. The method of balancing the links' weight values by repeatedly exposing the network to the input-output dataset for learning is called training.

Figure 2. The architecture of a Multi-layer feed-forward neural network with four inputs. ANN has various types of architectures. Multi-layer feed-forward neural networks have been widely used in cancer classification [40][41][42]. The architecture of a Multi-layer feed-forward neural network system is shown in Figure 2, consisting of one or more input layer and one or more hidden layer along with an output layer.

\section{The Proposed Artificial Neural Network Model with 15 Neurons in a Single Hidden Layer for Breast Cancer Classification}

In this section, an ANN model with 15 neurons in a single hidden layer is presented for breast cancer classification. The ANN model, which applied the Taguchi method [14] to determine the optimal number of neurons in the hidden layer for breast cancer classification, is called the Improved-ANN (IANN). The design of the proposed IANN model consists of six phases: data collection, data preprocessing, data loading using MATLAB simulation tool, data partitioning, 
278

279

280

281

282

283

284

285

286

287

288

289

290

291

292

293

294

295

296

297

298

299

300

301

302

303

304

305

306

307

308

309

310

311

312

313

314

315

316

ANN training and validation, and the application of the Taguchi method to determine the optimal number of neurons in the hidden layer of the ANN.

The first step is data collection. It involves the collection of the appropriate dataset from the data source and center for the purpose of research. In the second step (data preprocessing), all of the data features were prepared and filtered to remove noise in order to enhance the quality of the features that will be selected for the classification exercise. In the third stage, the Neural Network Pattern Recognition Tool of MATLAB (R2017b) simulation software [43] was used to load the dataset. Then, the dataset was divided into three categories: a training dataset, a validation dataset, and a testing dataset. A two-layer feed-forward neural network, Gradient Descent withSigmoid activation function and Softmax Output Neurons (patternnet), was used to classify the dataset with adequate neurons in the hidden layer. Multi-layer feed-forward neural network widely used for classification problem and it's able to achieved high classification performance. The most algorithm to train neural network is used gradient descent. Gradient descent is a way to minimize an objective function which assist to adapt the learning rate. The sigmoid activation function is a nonlinear function which can help the network learn complex data in hidden layer. The softmax function can able to handle multiple classes output. Basically, Softmax function is used for output layer. The number of neurons for the experiments was at least 10 (default value) because below that the performance was too low. It could be seen that, in the case of each training phase, the performance of the model increased gradually and peaked when the number of neurons in the hidden layer hit 15 . Further increasing the number of hidden layer neurons, from 16 to 20 neurons, the performance was observed to decrease. The number of neurons determined using the Taguchi method is detailed in Section 5.1 and Table 2. By this method, the number of hidden layer neurons was selected randomly and repeatedly until excellent accuracy was obtained for the validation set. The excellent performance of the validation set in this study was achieved via 15 neurons in the hidden layer, which was sufficient to construct a suitable ANN model for breast cancer classification when the number of output layer neurons was two, as shown in Figure 3.

Figure 3. Architecture of the proposed 15 neurons ANN classifier and two output layers.

Figure 4. The proposed Improved-ANN (IANN) cancer classification model.

Understanding the classification of breast cancer forms the first step in its containment and eradication worldwide. Cancer researchers have utilized various machine learning algorithms for cancer classification. The most significant benefit of ANN for the classification of the problem in multisource databases has been solved [45][46][47]. This study employs an ANN with 15 neurons in hidden layer for cancer classification. Figure 4 shows the conceptual framework for breast cancer classification using an ANN model with 15 neurons in its hidden layer.

\section{Experimental Results and Discussion}

Experimental Setup and Evolution Methods

Peer) Comput. Sci. reviewing PDF | (CS-2020:08:52384:1:1:NEW 23 Nov 2020) 
317 In this research, the experiment was conducted by using MATLAB Tool, WDBC dataset, Taguchi 318 Method and ANN Algorithm, implemented on a system with the following configuration: Core i7 319 GPU, Windows 10 with 8GB RAM and 1TB HDD. The proposed ANN model comprises a single 320 hidden layer with 15 neurons carefully selected using Taguchi method. Accuracy of the proposed

321

322

323

324

325

326

327

328

329

330

331

332

333

334

335

336

337

338

339

340

341

342

343

344

345

346

347

348

349

350

351

352

353

354

355

356

357

358 model was obtained directly from the confusion matrix using the formula:

$$
\text { Accuracy }=\frac{\mathrm{TP}+\mathrm{TN}}{\mathrm{TP}+\mathrm{TN}+\mathrm{FP}+\mathrm{FN}} \times 100
$$

\section{ANN Parameter Optimization Based on the Taguchi Method}

To build an appropriate classification model using ANN, parameter selection is one of the most significant steps. The performance and stability of an ANN is dictated by its selected parameters. The Taguchi method is mainly used for experimental parametric selection, owing to the concept of engineering and technology. For instance, this method can decrease the vast number of experiments and simultaneously analyze several parameters. For excellent performance and lowcost computations, this method performs well in the context of systematic and efficient designs [47]. A different partition for a dataset can be created to simplify the selection of a suitable dataset.

As per Section 3.1, the $\mathrm{L}_{21}$ Orthogonal array was used to determine the appropriate number of neurons in the hidden layer of the proposed IANN model. The number of neurons for the experiments ranges from 10 (the default value) to 20, as shown in Table 2. Also, different partitions of the dataset were utilized in order to determine a suitable partition for the breast cancer dataset. For the different partitions of the dataset, Taguchi Orthogonal array mechanism was implemented in the same way, where $\mathrm{N}=3$ is the number of design factors of dataset partitions. Therefore, the $\mathrm{L}_{4}$ Orthogonal array was used for data partition (for more details, see section 3.1, equation 1). The dataset was randomly divided into three partitions: training, validation, and testing. These partitions were utilized in different groups: first, $50 \%, 25 \%$, and $25 \%$; second, $60 \%, 20 \%$, and $20 \%$; third, $70 \%, 15 \%$, and $15 \%$; and fourth, $80 \%, 10 \%$, and $10 \%$.

Table 2. The Taguchi method for ANN to select the suitable number of neurons for breast cancer datasets.

It can be observed in Table 2 that the hidden layer parameter is crucial to the performance of the ANN. As established earlier, the experiments were conducted in four phases using different dataset partitions for each phase. For the first experiment, we partitioned the entire dataset into 50\% training, 25\% validation, and the remaining 25\% for testing. Based on the three partitions used, the highest accuracy for the validation dataset was realized when the number of neurons in a single hidden layer is 15 . In this experiment, the accuracy was found to be $93.90 \%$ for the validation dataset and $93.50 \%$ for the training dataset. However, when the number of neurons increased, the performance diminished significantly. This seemingly implied that when the number of neurons in the hidden layer increases further, the performance of the ANN model is negatively affected.

In the second experiment, when the partitioning of the dataset was changed to $60 \%, 20 \%$, and $20 \%$ for training, validation, and testing, respectively, the Taguchi method confirmed that the highest performance was maintained at 15 neurons in a single hidden layer. It is therefore clear from Table II that utilizing the aforementioned partitions significantly improves the performance of IANN model by $\sim 3.0 \%$.

Peer] Comput. Sci. reviewing PDF | (CS-2020:08:52384:1:1:NEW 23 Nov 2020) 
359

360

361

362

363

364

365

366

367

368

369

370

371

372

373

374

375

376

377

378

379

380

381

382

383

384

385

386

387

388

389

390

391

392

393

394

395

396

In the third experiment, a new partition for the dataset was introduced, with $70 \%$ data for training, $15 \%$ for validation, and $15 \%$ for testing. In this scenario, the highest accuracy for the validation dataset was determined to be $98.30 \%$, and that of the training dataset was $98.50 \%$ when the number of neurons in a single hidden layer was 15 using the Taguchi method.

Finally, in the fourth experiment, the partitioning of the dataset was $80 \%, 10 \%$, and $10 \%$ for training, validation, and testing, respectively. This study confirmed that the results declined from $98.30 \%$ for the validation dataset to $93.80 \%$ with $4.5 \%$ when compared with the third experiment. All of the experiments proved that, 15 neurons are sufficient for modeling a single hidden layer in the proposed IANN for breast cancer classification.

From the empirical results, this study posits that the optimum conditions for breast cancer classification are 15 neurons in a single hidden layer and the partitioning of the data into $70 \%$, $15 \%$, and $15 \%$ for training, validation, and testing sets, respectively. Thus, we built an IANN model with three layers: input, hidden, and output. In summary, there were 31 input neurons at the input layer and 2 output neurons at the output layer, while the number of neurons in a single hidden layer was 15.

\section{Breast Cancer Classification Results}

As established in Section 4, the suitable number of neurons for a single hidden layer based on the Taguchi is 15 . In addition, $70 \%$ of the dataset for training, $15 \%$ for validation, and $15 \%$ for testing is the optimal partitioning. The experiments were simulated on MATLAB (R2017b) software using Neural Network Toolbox for the implementation of the proposed model. The proposed IANN model has been tested using the WDBC dataset.

Table 3 shows the results from 30 different classification simulations using the WDBC dataset with their corresponding percentage errors. The average of the tests' accuracy was $98.80 \%$. This study confirmed that the results of the simulations were relatively similar, which could be due to the high stability resulting from the selection of suitable parameters for the IANN classifier.

Table 3. Classification accuracy of the proposed method based on 30 different runs.

This study used 569-sample datasets. Each data sample consists of 32 features, with a total of 18,208 data points distributed as follows: 11,424 data points for normal and 6784 data points for cancerous. These features were used to train and simulate the proposed IANN model. A hiddenlayer simple feed-forward neural network architecture is considered because our aim is to enhance the classification of breast cancer through optimal neuron selection in the hidden layer. The proposed IANN was tested with training, validation, and testing sets from the dataset. The network was adjusted based on the reported error during testing. Validation was used to simplify the network and halt training. Testing is ineffective in training; therefore, the performance of the network provides independent measures during and after training. A breast cancer dataset consisting of 569 samples was divided randomly into two groups: 399 samples $(70 \%)$ for training and 170 samples $(30 \%)$ for testing. The dataset for training was randomly divided into three

Peer) Comput. Sci. reviewing PDF | (CS-2020:08:52384:1:1:NEW 23 Nov 2020) 
397

398

399

400

401

402

403

404

405

406

407

408

409

410

411

412

413

414

415

416

417

418

419

420

421

422

423

424

425

426

427

428

429

430

431

432

433

434

groups: out of 399 samples $279(70 \%)$ samples for training, and $60(15 \%)$ samples each for testing and validation respectively.

Figures 5-11 show the IANN training performance, training state performance, error histogram, performance confusion matrix, ROC curve and testing dataset results for breast cancer, respectively.

Figure 5. IANN training performance.

It can be seen in Figure 5 that the IANN training performance plot at the beginning of the training of cross-entropy resulted in the maximum error. The proposed system reported the best performance at epoch 20 iterations and an exact cross-entropy of 0.031613 .

Figure 6 shows the network training state performance at epoch 26, when the gradient is 0.032875 . The network halts the training session because its generalization stops improving.

Error histograms for training, validation, and testing data are shown with 20 bins in Figure 7. The experimental results shown in error histogram of Figure 7 indicate that, the proposed system can handle the dataset used successfully, since the error is close to zero.

Figure 6. Training state performance.

Figure 7. Error histogram.

To evaluate the accuracy, a confusion matrix was used for all partitions of the dataset. A confusion matrix is a two-dimensional array, $r \times r$ (where $r$ is the number of classes). Figure 8 shows the MATLAB output representation of training dataset confusion matrix performance after 10 -fold cross-validation. In Figure 8, the first two rows and first two columns represent the actual confusion matrix. The third row and third column are the summary of percentage accuracy, and sensitivity and specificity, respectively. Row one, column one is the true positives (TP); row one, column two is the false negatives (FN); row two, column one is the false positives (FP); and row two, column two is the true negatives, respectively. All false positives (FP) are Type I errors, while all false negative (FN) are Type II errors generated by different classifiers.

The first two diagonal cells of the confusion matrix demonstrate the correct classification number and percentage accuracy of the trained network. The normal biopsy results are 247 , which were correctly classified to represent $61.90 \%$ of the total 399 biopsies. On the other hand, the cancerous (malignant) biopsy results show 146 correctly classified tumors, representing 36.60\% of the total biopsies. The experiment also revealed that three of the cancerous biopsies were incorrectly classified as normal, representing $0.8 \%$ of the total biopsy dataset, while three of the normal biopsies were incorrectly classified as cancerous, also representing $0.8 \%$ of the total biopsy data. The total result of 250 normal revealed that $98.80 \%$ was correct, and $1.20 \%$ incorrect. The total result of 149 cancerous was $98.00 \%$ correct and $2.00 \%$ incorrect. The total result of 250 normal cases was $98.80 \%$ correctly classified as normal, and $1.2 \%$ classified as cancerous. Out of 
435149 cancerous cases, $98.00 \%$ were correctly classified as cancer, and $2.00 \%$ classified as normal. 436 The confusion matrix plots with $98.50 \%$ accuracy show that this system performed well and had $4371.40 \%$ misclassification during its training stage from the proposed IANN.

438

439

440

441

442

443

444

445

446

447

448

449

450

451

452

453

454

455

456

457

458

459

460

461

462

463

464

465

466

467

468

469

Figure 8. Performance confusion matrix.

Figure 9. Receiver operating characteristic (ROC) curve.

The neural network training performance with receiver operating characteristic (ROC) plot is shown in Figure 9. The ROC plot represents the performance of the binary classification system when the discrimination threshold fluctuates. The graph is formed by plotting the true positive rate (TPR) against the false positive rate (FPR). From ROC plot, it can be seen that the NN's performance increases in response to the number of iterations. A perfect classification result was evident at 26 iterations. This shows that every class achieved perfect classification accuracy. Iteration 26 is the optimal iteration for the proposed model where the IANN performed at its peak.

After completing the training session, we tested the network for accurate classification, using $30 \%$ of the test dataset of breast cancer. Figure 10 shows the testing accuracy, otherwise known as the classification accuracy from the proposed IANN, to be rather high.

Figure 10. Test dataset result of breast cancer.

Figure 10 shows the experimental results with a test dataset of breast cancer: normal versus malignant tumor is correctly classified at $98.8 \%$ accuracy for the test case.

This study computed the receiver operating curve (ROC) and the results are shown in Figure 11. The ROC curve indicates TPR and FPR at different edge settings of the network, with better results arising from the proposed system. The IANN, after training, validation, and testing, achieved $98.8 \%$ correct classification for two classes: normal and cancerous (malignant). The area under the curve was large.

Table 4 shows the model performance obtained from the confusion matrix shown in Figures 8 and 10. In Table 4, the F-Score for the training dataset is 98.4\%, while the F-Score for the testing dataset is $98.8 \%$.

Table 4. Model performance based on confusion matrix after 10-fold cross-validation.

\section{Discussion of Findings and Implications}

Table 5 shows the performance of multiple machine learning algorithms relative to the proposed IANN model. Performance comparison was realized via MATLAB Classification learner tool- 
470

471

472

473

474

475

476

477

478

479

480

481

482

483

484

485

486

487

488

489

490

491

492

493

494

495

496

497

498

499

500

501

502

503

504

505

506

507

508

staking for the breast cancer dataset. Although the results obtained with other machine learning algorithms are equally good, the result reported by our model is optimal. It can be surmised that the proposed IANN outperforms the existing machine learning models in classifying breast cancer.

Table 5. Performance comparison between different classifiers and the proposed method in breast cancer classification.

Table 6. Comparison of breast cancer classification performance between existing methods in the literature and the proposed method.

Table 6 indicates the superiority of this study in the context of cancer classification based on the same dataset but different methods for classification. Among the previous studies tabulated in Table 6, a lower classification accuracy of $92.98 \%$ was achieved by Xue et al. [31]. These authors proposed the PSO technique with novel initialization and updated mechanisms hybridized with a KNN classifier and 10-fold cross-validation to maximize the classification performance of the WDBC datasets and dataset were partitioned for training 70\% and 30\% for testing. Quy et al. [36] achieved the best classification accuracy of 98.24\% using APSO-NN. For the NN used 20 neurons in a single hidden layer. The WDBC dataset was used and partitioned $70 \%$ for training and 30\% for testing. Abdar et al. [49] used the WDBC dataset for the experiment and achieved a classification accuracy of $98.07 \%$ using SV-NB-3-MetaClassifiers with a K-fold cross-validation technique. The performance is quite good. Nekkaa et al. [32] used a memetic algorithm (MA) with support vector machine (SVM) to address the classification problem. The authors used particular datasets to compare certain popular classifiers for the data classification task. The model achieved 97.85\% accuracy using WDBC datasets. Beside, Salama et al. [27] achieved a classification accuracy of $97.70 \%$ using sequential minimal optimization (SMO) technique with a confusion matrix based on the 10-fold cross-validation method. Amrane et al. [48] used the KNN and Naive Bayes (NB) classifier machine learning technique for breast cancer classification. The author partitioned the dataset $60 \%$ for training and $40 \%$ for tesing. From KNN classifier achieved the highest classification accuracy of $97.51 \%$ which is not so high. Aalaei et al. [5] used a GA-based classifier with ANN and reported a classification accuracy of $96.50 \%$ by making use of a single hidden layer with 5-neurons and 2-neurons in output layer, and the dataset partitioned of $80 \%$ for training and $20 \%$ for test data.

From the analysis of the results presented in Tables 5 and 6, it is obvious that the proposed method outperformed the other methods due to it realizing a classification accuracy of $98.80 \%$ — an improvement of $0.56 \%$ compared to Quy et al. [36], which has the best performance among the existing methods. This could be due to the use of the Taguchi method for selecting the optimal number of neurons in a single hidden layer of ANN and suitable percentage data partition. Our results also show that the proposed method is more stable and reliable than existing classification models. By implication, incorporating IANN into breast cancer prediction could enable timely and accurate prediction of breast cancer, thereby helping medical practitioners to make the most appropriate decisions on breast cancer treatment. Table 7 (where $K=10$ ) shows the 10 -fold cross 
509 validation experimental performance which was conducted to validate the results shown in Table 5105 and 6.

Table 7. 10-fold cross-validation experimental performance.

\section{Conclusions}

513 In breast cancer diagnosis, differentiating between normal and malignant tumors is one of the 514 challenges faced by physicians. In order to tackle this problem, ANN is incorporated in the CAD

515 system for the binary classification of breast cancer datasets into normal/malignant tumors. The 516 main purpose of this research is to enhance the classification accuracy to improve image-based 517 diagnosis. The proposed IANN utilized the Taguchi method to optimize the hidden layer parameter 518 of the ANN model. The experimental results proved that 15 is the optimal number of neurons for 519 a single hidden layer of ANN, and can enhance the classification accuracy for the training, testing, 520 and validation of selected datasets.

521 In this paper, the proposed IANN method reported $98.80 \%$ classification accuracy for breast 522 cancer dataset classification. Additionally, 10-folds cross validation experiments performed 523 (shown in Table 7) also confirmed the efficiency of the proposed IANN model with an average 524 accuracy of $98.7 \%$ on testing dataset. The empirical results, presented in both tabular and graphical 525 form, proved that the proposed IANN greatly enhanced the overall classification performance by 526 differentiating between normal and malignant tumors for superior breast cancer diagnosis. This 527 study confirmed that the use of the Taguchi method with ANN improved classification 528 performance relative to existing breast cancer classification methods. While the results are 529 promising, future research can focus on feature selection to reduce the computational discrepancy. 530 Also, future studies should focus on the practical implications of Type I (FP) and Type II (FN) 531 error in breast cancer classification. Meanwhile, an express medical test for breast cancer 532 classification has often been designed in such a way that Type I error is given precedence. This is 533 done to prevent a breast cancer patient from going about without the knowledge of having a 534 malignant tumor.

535

536

537

538

539

540

541

542

543

544

545

\section{Acknowledgments}

The grant for this research (Universiti Kebangsaan Malaysia (UKM), UKM Grant Code: GGP2019-023) is acknowledged, as this support played a vital role in the successful accomplishment of the research.

\section{References}

1. "Breast Cancer - Symptoms \&amp; Treatment Resources - Imaginis." [Online]. Available: http://www.imaginis.com/breast-cancer-resource-center. [Accessed: 24-Apr-2019].

2. S. Sahran, D. Albashish, A. Abdullah, N. A. Shukor, and S. Hayati Md Pauzi, "Absolute cosine-based SVM-RFE feature selection method for prostate histopathological grading," Artif. Intell. Med., Apr. 2018. 
546 3. D. Albashish, S. Sahran, A. Abdullah, N. AbdShukor, and S. Hayati Md Pauz, "Ensemble

547

548

549

550

551

552

553

554

555

556

557

558

559

560

561

562

563

564

565

566

567

568

569

570

571

572

573

574

575

576

577

578

579

580

581

582

583

584

585

586

587

588

589

590

591 Learning of Tissue Components for Prostate Histopathology Image Grading," Eng. Inf. Technol. (IJASEIT)., vol. 6, no. 6, pp. 1132-1138, 2016.

4. M. Xi, J. Sun, L. Liu, F. Fan, and X. Wu, "Cancer Feature Selection and Classification Using a Binary Quantum-Behaved Particle Swarm Optimization and Support Vector Machine," Comput. Math. Methods Med., vol. 2016, pp. 1-9, 2016.

5. S. Aalaei, H. Shahraki, A. Rowhanimanesh, and S. Eslami, "Feature selection using genetic algorithm for breast cancer diagnosis: experiment on three different datasets.," Iran. J. Basic Med. Sci., vol. 19, no. 5, pp. 476-82, May 2016.

6. M. A. Rahman and R. C. Muniyandi, "Review of GPU implementation to process of RNA sequence on cancer," Informatics Med. Unlocked, vol. 10, pp. 17-26, Jan. 2018.

7. D. Tomar and S. Agarwal, "Hybrid feature selection based weighted least squares twin support vector machine approach for diagnosing breast cancer, hepatitis, and diabetes," Adv. Artif. Neural Syst., vol. 2015, no. article.1, 2015.

8. N. Elkhani and R. C. Muniyandi, "Membrane computing inspired feature selection model for microarray cancer data," Intell. Data Anal., vol. 21, no. S1, pp. S137-S157, Apr. 2017.

9. J. Dheeba, N. Albert Singh, and S. Tamil Selvi, "Computer-aided detection of breast cancer on mammograms: A swarm intelligence optimized wavelet neural network approach," J. Biomed. Inform., vol. 49, pp. 45-52, Jun. 2014.

10. Jafari-Marandi, Ruholla, Samaneh Davarzani, Maryam Soltanpour Gharibdousti, and Brian K. Smith. "An optimum ANN-based breast cancer diagnosis: Bridging gaps between ANN learning and decision-making goals." Applied Soft Computing 72 (2018): 108-120.

11. M. A. Rahman and R. C. Muniyandi, "Feature selection from colon cancer dataset for cancer classification using Artificial Neural Network," Int. J. Adv. Sci. Eng. Inf. Technol., vol. 8, no. 4-2, p. 1387, Sep. 2018.

12. S. Karsoliya, "Approximating number of hidden layer neurons in multiple hidden layer BPNN architecture,” Int. J. Eng. Trends Technol., vol. 3, no. 6, pp. 714-717, 2012.

13. G. Panchal, A. Ganatra, ... Y. K.-I. J. of, and U. 2011, "Behaviour analysis of multilayer perceptronswith multiple hidden neurons and hidden layers," Int. J. Comput. Theory Eng., vol. 3, no. 2, pp. 332-337, 2011.

14. Y. Wu and A. Wu, Taguchi Methods for robust design. ASME Press, 2000.

15. N. S. Jaddi, S. Abdullah, and A. R. Hamdan, "Taguchi-Based Parameter Designing of Genetic Algorithm for Artificial Neural Network Training," in 2013 International Conference on Informatics and Creative Multimedia, 2013, pp. 278-281.

16. J. F. C. Khaw, B. S. Lim, and L. E. N. Lim, "Optimal design of neural networks using the Taguchi method," Neurocomputing, vol. 7, no. 3, pp. 225-245, Apr. 1995.

17. G. E. Peterson, D. C. St. Clair, S. R. Aylward, and W. E. Bond, "Using Taguchi's method of experimental design to control errors in layered perceptrons," IEEE Trans. Neural Networks, vol. 6, no. 4, pp. 949-961, Jul. 1995.

18. S. M. Yang and G. S. Lee, "Neural Network Design by Using Taguchi Method," J. Dyn. Syst. Meas. Control, vol. 121, no. 3, p. 560, Sep. 1999.

19. M. S. Packianather, P. R. Drake, and H. Rowlands, "Optimizing the parameters of multilayered feedforward neural networks through Taguchi design of experiments," Qual. Reliab. Eng. Int., vol. 16, no. 6, pp. 461-473, Nov. 2000.

20. Y.-S. Kim and B.-J. Yum, "Robust design of multilayer feedforward neural networks: an experimental approach," Eng. Appl. Artif. Intell., vol. 17, no. 3, pp. 249-263, Apr. 2004.

Peer) Comput. Sci. reviewing PDF | (CS-2020:08:52384:1:1:NEW 23 Nov 2020) 
592

593

594

595

596

597

598

599

600

601

602

603

604

605

606

607

608

609

610

611

612

613

614

615

616

617

618

619

620

621

622

623

624

625

626

627

628

629

630

631

632

633

634

635

636

637

21. A. Tortum, N. Yayla, C. Çelik, and M. Gökdağ, "The investigation of model selection criteria in artificial neural networks by the Taguchi method," Phys. A Stat. Mech. its Appl., vol. 386, no. 1, pp. 446-468, Dec. 2007.

22. M. Bashiri and A. Farshbaf Geranmayeh, "Tuning the parameters of an artificial neural network using central composite design and genetic algorithm," Sci. Iran., vol. 18, no. 6, pp. 1600-1608, Dec. 2011.

23. J.-R. Jung and B.-J. Yum, "Artificial neural network-based approach for dynamic parameter design,” Expert Syst. Appl., vol. 38, no. 1, pp. 504-510, Jan. 2011.

24. N. Becherer, J. Pecarina, S. Nyk1, and K. Hopkinson, "Improving optimization of convolutional neural networks through parameter fine-tuning," Neural Comput. Appl., pp. 111, Nov. 2017.

25. B. Zheng, S. W. Yoon, and S. S. Lam, "Breast cancer diagnosis based on feature extraction using a hybrid of K-means and support vector machine algorithms," Expert Syst. Appl., vol. 41, no. 4, pp. 1476-1482, Mar. 2014.

26. H. H. Örkcü and H. Bal, "Comparing performances of backpropagation and genetic algorithms in the data classification," Expert Syst. Appl., vol. 38, no. 4, pp. 3703-3709, Apr. 2011.

27. G. Salama and M. Z. Abdelhalim, M, "Breast cancer diagnosis on three different datasets using multi-classifiers," Int. J. Comput. Inf. Technol., vol. 1, no. 1, 2012.

28. H. Malmir, F. Farokhi, and R. Sabbaghi-Nadooshan, "Optimization of data mining with evolutionary algorithms for cloud computing application," in ICCKE 2013, 2013, pp. 343347.

29. H. Koyuncu and R. Ceylan, "Artificial neural network based on rotation forest for biomedical pattern classification," in 2013 36th International Conference on Telecommunications and Signal Processing (TSP), 2013, pp. 581-585.

30. V. Chaurasia and S. Pal, "A Novel Approach for Breast Cancer Detection Using Data Mining Techniques,” Int. J. Innov. Res. Comput. Commun. Eng., vol. 2, no. 1, p. pp.17, Jun. 2017.

31. B. Xue, M. Zhang, and W. N. Browne, "Particle swarm optimisation for feature selection in classification: Novel initialisation and updating mechanisms," Appl. Soft Comput., vol. 18, pp. 261-276, May 2014.

32. M. Nekkaa and D. Boughaci, "A memetic algorithm with support vector machine for feature selection and classification," Memetic Comput., vol. 7, no. 1, pp. 59-73, Mar. 2015.

33. Idri, Ali, Mohamed Hosni, and Ibtissam Abnane. "Assessing the impact of parameters tuning in ensemble based breast Cancer classification." Health and Technology 10, no. 5 (2020): 1239-1255.

34. Jijitha, S., and Thangavel Amudha. "Breast Cancer Prognosis Using Machine Learning Techniques and Genetic Algorithm: Experiment on Six Different Datasets." In Evolutionary Computing and Mobile Sustainable Networks, pp. 703-711. Springer, Singapore. 01 August 2020.

35. Thiyagarajan,S., T. Chakravarthy, and PV Arivoli. "Diagnosing Breast Cancer with Machine Learning Algorithms".Int J Life Sci Pharma Res. ISSN 2250 - 0480; SP-07; 23rd January 2020: 42. "International Conference on Cancer Research" 2020.

36. Quy, Hoang Duc, Cao Van Kien, Ho Pham Huy Anh, and Nguyen Ngoc Son. "Machine Learning-Based Evolutionary Neural Network Approach Applied in Breast Cancer Tumor Classification." In International Conference on Green Technology and Sustainable Development, pp. 72-83. Springer, Cham, 2020. 
638

639

640

641

642

643

644

645

646

647

648

649

650

651

652

653

654

655

656

657

658

659

660

661

662

663

664

665

666

667

668

669
37. M. Pobiruchin, S. Bochum, U. M. Martens, M. Kieser, and W. Schramm, "A method for using real world data in breast cancer modeling," J. Biomed. Inform., vol. 60, pp. 385-394, Apr. 2016

38. L.-Y. Chuang, C.-S. Yang, K.-C. Wu, and C.-H. Yang, "Correlation-based Gene Selection and Classification Using Taguchi-BPSO,” Methods Inf. Med., vol. 49, no. 3, pp. 254-268, Jan. 2010.

39. C.-H. Yang, C.-C. Huang, K.-C. Wu, and H.-Y. Chang, "A Novel GA-Taguchi-Based Feature Selection Method," Springer, Berlin, Heidelberg, 2008, pp. 112-119.

40. O. Alesawy and R. C. Muniyandi, "Elliptic Curve Diffie-Hellman Random Keys Using Artificial Neural Network and Genetic Algorithm for Secure Data over Private Cloud," Inf. Technol. J., vol. 15, no. 3, pp. 77-83, Jun. 2016.

41. J. Hopfield, “Artificial neural networks," IEEE Circuits Devices Mag., vol. 4, no. 5, 1988.

42. G. Bebis and M. Georgiopoulos, "Feed-forward neural networks," IEEE Potentials, vol. 13, no. $4,1994$.

43. The Math Works Inc, "MATLAB 2017b," Natick, Massachusetts United States.

44. B. Cai and X. Jiang, "A novel artificial neural network method for biomedical prediction based on matrix pseudo-inversion,” J. Biomed. Inform., vol. 48, pp. 114-121, Apr. 2014.

45. M. Wadhonkar, P. Tijare, S. S.- Integration, and U. 2014, "Artificial Neural Network Approach for Classification of Heart Disease Dataset," ijaiem.org, vol. 3, no. 4, pp. 388-392, 2014.

46. Rahman, Md Akizur, and Ravie Chandren Muniyandi. "An Enhancement in Cancer Classification Accuracy Using a Two-Step Feature Selection Method Based on Artificial Neural Networks with 15 Neurons." Symmetry 12, no. 2 (2020): 271.

47. M.-L. Huang, Y.-H. Hung, W. M. Lee, R. K. Li, and B.-R. Jiang, "SVM-RFE based feature selection and Taguchi parameters optimization for multiclass SVM classifier," ScientificWorldJournal., vol. 2014, p. 795624, Jan. 2014.

48. M. Amrane, S. Oukid, I. Gagaoua, and T. Ensari, "Breast cancer classification using machine learning," in 2018 Electric Electronics, Computer Science, Biomedical Engineerings' Meeting (EBBT), 2018, pp. 1-4.

49. Abdar, Moloud, Mariam Zomorodi-Moghadam, Xujuan Zhou, Raj Gururajan, Xiaohui Tao, Prabal D. Barua, and Rashmi Gururajan. "A new nested ensemble technique for automated diagnosis of breast cancer." Pattern Recognition Letters 132 (2020): 123-131. 


\section{Table $\mathbf{1}$ (on next page)}

Table I. Orthogonal array (OA) 


\begin{tabular}{ccccccccccccccccccccc}
1 & \multicolumn{110}{c}{ Table I. Orthogonal array $(\mathrm{OA})$} \\
\hline RUN & $\mathbf{F 1}$ & $\mathbf{F 2}$ & $\mathbf{F 3}$ & $\mathbf{F 4}$ & $\mathbf{F 5}$ & $\mathbf{F 6}$ & $\mathbf{F 7}$ & $\mathbf{F 8}$ & $\mathbf{F 9}$ & $\mathbf{F 1 0}$ & $\mathbf{F 1 1}$ & $\mathbf{F 1 2}$ & $\mathbf{F 1 3}$ & $\mathbf{F 1 4}$ & $\mathbf{F 1 5}$ & $\mathbf{F 1 6}$ & $\mathbf{F 1 7}$ & $\mathbf{F 1 8}$ & $\mathbf{F 1 9}$ & $\mathbf{F 2 0}$ \\
\hline $\mathbf{1}$ & $\mathbf{1}$ & 0 & 0 & 0 & 0 & 0 & 0 & 0 & 0 & 0 & 0 & 0 & 0 & 0 & 0 & 0 & 0 & 0 & 0 & 0 \\
\hline $\mathbf{2}$ & 0 & $\mathbf{1}$ & 0 & 0 & 0 & 0 & 0 & 0 & 0 & 0 & 0 & 0 & 0 & 0 & 0 & 0 & 0 & 0 & 0 & 0 \\
\hline $\mathbf{3}$ & 0 & 0 & $\mathbf{1}$ & 0 & 0 & 0 & 0 & 0 & 0 & 0 & 0 & 0 & 0 & 0 & 0 & 0 & 0 & 0 & 0 & 0 \\
\hline $\mathbf{4}$ & 0 & 0 & 0 & $\mathbf{1}$ & 0 & 0 & 0 & 0 & 0 & 0 & 0 & 0 & 0 & 0 & 0 & 0 & 0 & 0 & 0 & 0 \\
\hline $\mathbf{5}$ & 0 & 0 & 0 & 0 & $\mathbf{1}$ & 0 & 0 & 0 & 0 & 0 & 0 & 0 & 0 & 0 & 0 & 0 & 0 & 0 & 0 & 0 \\
\hline $\mathbf{6}$ & 0 & 0 & 0 & 0 & 0 & $\mathbf{1}$ & 0 & 0 & 0 & 0 & 0 & 0 & 0 & 0 & 0 & 0 & 0 & 0 & 0 & 0 \\
\hline $\mathbf{7}$ & 0 & 0 & 0 & 0 & 0 & 0 & $\mathbf{1}$ & 0 & 0 & 0 & 0 & 0 & 0 & 0 & 0 & 0 & 0 & 0 & 0 & 0 \\
\hline $\mathbf{8}$ & 0 & 0 & 0 & 0 & 0 & 0 & 0 & $\mathbf{1}$ & 0 & 0 & 0 & 0 & 0 & 0 & 0 & 0 & 0 & 0 & 0 & 0 \\
\hline $\mathbf{9}$ & 0 & 0 & 0 & 0 & 0 & 0 & 0 & 0 & $\mathbf{1}$ & 0 & 0 & 0 & 0 & 0 & 0 & 0 & 0 & 0 & 0 & 0 \\
\hline $\mathbf{1 0}$ & 0 & 0 & 0 & 0 & 0 & 0 & 0 & 0 & 0 & $\mathbf{1}$ & 0 & 0 & 0 & 0 & 0 & 0 & 0 & 0 & 0 & 0 \\
\hline $\mathbf{1 1}$ & 0 & 0 & 0 & 0 & 0 & 0 & 0 & 0 & 0 & 0 & $\mathbf{1}$ & 0 & 0 & 0 & 0 & 0 & 0 & 0 & 0 & 0 \\
\hline $\mathbf{1 2}$ & 0 & 0 & 0 & 0 & 0 & 0 & 0 & 0 & 0 & 0 & 0 & $\mathbf{1}$ & 0 & 0 & 0 & 0 & 0 & 0 & 0 & 0 \\
\hline $\mathbf{1 3}$ & 0 & 0 & 0 & 0 & 0 & 0 & 0 & 0 & 0 & 0 & 0 & 0 & $\mathbf{1}$ & & 0 & 0 & 0 & 0 & 0 & 0 \\
\hline $\mathbf{1 4}$ & 0 & 0 & 0 & 0 & 0 & 0 & 0 & 0 & 0 & 0 & 0 & 0 & 0 & $\mathbf{1}$ & 0 & 0 & 0 & 0 & 0 & 0 \\
\hline $\mathbf{1 5}$ & 0 & 0 & 0 & 0 & 0 & 0 & 0 & 0 & 0 & 0 & 0 & 0 & 0 & 0 & $\mathbf{1}$ & 0 & 0 & 0 & 0 & 0 \\
\hline $\mathbf{1 6}$ & 0 & 0 & 0 & 0 & 0 & 0 & 0 & 0 & 0 & 0 & 0 & 0 & 0 & 0 & 0 & $\mathbf{1}$ & 0 & 0 & 0 & 0 \\
\hline $\mathbf{1 7}$ & 0 & 0 & 0 & 0 & 0 & 0 & 0 & 0 & 0 & 0 & 0 & 0 & 0 & 0 & 0 & 0 & $\mathbf{1}$ & 0 & 0 & 0 \\
\hline $\mathbf{1 8}$ & 0 & 0 & 0 & 0 & 0 & 0 & 0 & 0 & 0 & 0 & 0 & 0 & 0 & 0 & 0 & 0 & 0 & $\mathbf{1}$ & 0 & 0 \\
\hline $\mathbf{1 9}$ & 0 & 0 & 0 & 0 & 0 & 0 & 0 & 0 & 0 & 0 & 0 & 0 & 0 & 0 & 0 & 0 & 0 & 0 & $\mathbf{1}$ & 0 \\
\hline $\mathbf{2 0}$ & 0 & 0 & 0 & 0 & 0 & 0 & 0 & 0 & 0 & 0 & 0 & 0 & 0 & 0 & 0 & 0 & 0 & 0 & 0 & $\mathbf{1}$ \\
\hline $\mathbf{2 1}$ & 0 & 0 & 0 & 0 & 0 & 0 & 0 & 0 & 0 & 0 & 0 & 0 & 0 & 0 & 0 & 0 & 0 & 0 & $\mathbf{1}$ & 0 \\
\hline $\mathbf{2}$ & & & & & & & & & & & & & & & & &
\end{tabular}




\section{Table 2 (on next page)}

Table II. The Taguchi method for ANN to select the suitable number of neurons for breast cancer datasets 
Table II. The Taguchi method for ANN classification to select the suitable number of neurons for breast cancer datasets.

\begin{tabular}{|c|c|c|c|c|c|}
\hline \multirow{2}{*}{$\begin{array}{c}\text { First } \\
\text { Number of } \\
\text { neurons selected } \\
\text { by } \\
\text { Taguchi method }\end{array}$} & \multirow{2}{*}{$\begin{array}{l}\text { Training } \\
\text { Accuracy } \\
\quad(\%)\end{array}$} & \multirow{2}{*}{$\begin{array}{c}\text { Validation } \\
\text { Accuracy } \\
(\%)\end{array}$} & \multicolumn{3}{|c|}{ Data Partitioning } \\
\hline & & & $\begin{array}{c}\text { Training } \\
(\%)\end{array}$ & $\begin{array}{c}\text { Validation } \\
(\%)\end{array}$ & $\begin{array}{c}\text { Testing } \\
(\%)\end{array}$ \\
\hline 10 & 89.7 & 89.4 & \multirow{11}{*}{50} & \multirow{11}{*}{25} & \multirow{11}{*}{25} \\
\hline 11 & 89.9 & 90.8 & & & \\
\hline 12 & 90.8 & 92.2 & & & \\
\hline 13 & 91.5 & 92.5 & & & \\
\hline 14 & 92.7 & 92.8 & & & \\
\hline 15 & 93.5 & 93.9 & & & \\
\hline 16 & 93.0 & 93.4 & & & \\
\hline 17 & 92.8 & 91.9 & & & \\
\hline 18 & 92.1 & 92.3 & & & \\
\hline 19 & 91.2 & 91.7 & & & \\
\hline 20 & 90.9 & 91.3 & & & \\
\hline Second & \multirow{3}{*}{$\begin{array}{l}\text { Training } \\
\text { Accuracy } \\
\quad(\%)\end{array}$} & \multirow{3}{*}{$\begin{array}{c}\text { Validation } \\
\text { Accuracy (\%) }\end{array}$} & \multirow{2}{*}{\multicolumn{3}{|c|}{ Data Partition }} \\
\hline Number of & & & & & \\
\hline $\begin{array}{l}\text { neurons selected } \\
\text { by Taguchi } \\
\text { method }\end{array}$ & & & $\begin{array}{l}\text { Training } \\
\quad(\%)\end{array}$ & $\begin{array}{l}\text { Validation } \\
(\%)\end{array}$ & $\begin{array}{c}\text { Testing } \\
(\%)\end{array}$ \\
\hline 20 & 90.7 & 91.1 & \multirow{11}{*}{60} & \multirow{11}{*}{20} & \multirow{11}{*}{20} \\
\hline 19 & 90.1 & 90.8 & & & \\
\hline 18 & 91.9 & 91.2 & & & \\
\hline 17 & 93.7 & 92.8 & & & \\
\hline 16 & 95.2 & 94.7 & & & \\
\hline 15 & 96.6 & 96.2 & & & \\
\hline 14 & 96.1 & 96.2 & & & \\
\hline 13 & 95.8 & 95.6 & & & \\
\hline 12 & 95.4 & 95.3 & & & \\
\hline 11 & 93.8 & 94.2 & & & \\
\hline 10 & 92.3 & 93.0 & & & \\
\hline Third & \multirow{3}{*}{$\begin{array}{l}\text { Training } \\
\text { Accuracy } \\
\quad(\%)\end{array}$} & \multirow{3}{*}{$\begin{array}{c}\text { Validation } \\
\text { Accuracy }(\%)\end{array}$} & \multirow{2}{*}{\multicolumn{3}{|c|}{ Data Partition }} \\
\hline Number of & & & & & \\
\hline $\begin{array}{c}\text { neurons selected } \\
\text { by Taguchi } \\
\text { method }\end{array}$ & & & $\begin{array}{l}\text { Training } \\
\quad(\%)\end{array}$ & $\begin{array}{c}\text { Validation } \\
(\%)\end{array}$ & $\begin{array}{c}\text { Testing } \\
(\%)\end{array}$ \\
\hline 10 & 93.6 & 93.1 & & & \\
\hline 11 & 94.5 & 94.3 & & & \\
\hline 12 & 95.7 & 94.9 & & & \\
\hline 13 & 97.2 & 96.8 & & & \\
\hline 14 & 98.0 & 97.5 & & & \\
\hline
\end{tabular}




\begin{tabular}{|c|c|c|c|c|c|}
\hline 15 & 98.5 & 98.3 & \multirow[t]{6}{*}{70} & \multirow[t]{6}{*}{15} & \multirow[t]{6}{*}{15} \\
\hline 16 & 98.3 & 98.2 & & & \\
\hline 17 & 97.8 & 97.6 & & & \\
\hline 18 & 97.1 & 97.1 & & & \\
\hline 19 & 96.8 & 96.3 & & & \\
\hline 20 & 96.2 & 96.3 & & & \\
\hline Fourth & \multirow{3}{*}{$\begin{array}{l}\text { Training } \\
\text { Accuracy } \\
\quad(\%)\end{array}$} & \multirow{3}{*}{$\begin{array}{c}\text { Validation } \\
\text { Accuracy (\%) }\end{array}$} & \multirow{2}{*}{\multicolumn{3}{|c|}{ Data Partition }} \\
\hline Number of & & & & & \\
\hline $\begin{array}{c}\text { neurons selected } \\
\text { by Taguchi } \\
\text { method }\end{array}$ & & & $\begin{array}{c}\text { Training } \\
(\%)\end{array}$ & $\begin{array}{c}\text { Validation } \\
(\%)\end{array}$ & $\begin{array}{c}\text { Testing } \\
(\%)\end{array}$ \\
\hline 20 & 93.4 & 93.1 & \multirow{11}{*}{80} & \multirow{11}{*}{10} & \multirow{11}{*}{10} \\
\hline 19 & 92.8 & 93.2 & & & \\
\hline 18 & 92.2 & 92.9 & & & \\
\hline 17 & 93.1 & 92.7 & & & \\
\hline 16 & 93.9 & 92.4 & & & \\
\hline 15 & 94.7 & 93.8 & & & \\
\hline 14 & 94.2 & 93.4 & & & \\
\hline 13 & 93.9 & 93.8 & & & \\
\hline 12 & 93.2 & 93.6 & & & \\
\hline 11 & 93.0 & 92.5 & & & \\
\hline 10 & 92.9 & 92.4 & & & \\
\hline
\end{tabular}

3 


\section{Table 3 (on next page)}

Table III . Classification accuracy of the proposed method based on 30 different runs 
Table III. Classification accuracy of the proposed method based on 30 different runs

\begin{tabular}{|c|c|c|c|c|}
\hline $\begin{array}{l}\text { Number of } \\
\text { Experiments }\end{array}$ & $\begin{array}{c}\text { Training } \\
\text { Accuracy } \\
(\%)\end{array}$ & $\begin{array}{c}\text { Validation } \\
\text { Accuracy } \\
(\%)\end{array}$ & $\begin{array}{c}\text { Testing } \\
\text { Accuracy } \\
(\%)\end{array}$ & $\begin{array}{c}\text { Percent } \\
\text { Error }\end{array}$ \\
\hline 1 & 97.7 & 96.5 & 98.7 & 1.3 \\
\hline 2 & 98.5 & 98.7 & 98.8 & 1.2 \\
\hline 3 & 98.9 & 97.9 & 99.2 & 0.8 \\
\hline 4 & 98.2 & 98.7 & 98.5 & 1.5 \\
\hline 5 & 97.7 & 97.2 & 98.6 & 1.4 \\
\hline 6 & 98.6 & 98.6 & 98.8 & 1.2 \\
\hline 7 & 98.8 & 98.5 & 99.2 & 0.8 \\
\hline 8 & 98.4 & 98.2 & 98.4 & 1.6 \\
\hline 9 & 98.7 & 98.7 & 99.2 & 0.8 \\
\hline 10 & 98.5 & 98.5 & 98.8 & 1.2 \\
\hline 11 & 98.2 & 98.2 & 98.8 & 1.2 \\
\hline 12 & 98.8 & 98.8 & 99.2 & 0.8 \\
\hline 13 & 98.3 & 98.0 & 98.6 & 1.4 \\
\hline 14 & 98.5 & 98.3 & 98.8 & 1.2 \\
\hline 15 & 98.3 & 98.5 & 98.7 & 1.3 \\
\hline 16 & 98.5 & 98.5 & 98.9 & 1.1 \\
\hline 17 & 98.6 & 98.5 & 99.2 & 0.8 \\
\hline 18 & 98.8 & 98.5 & 99.2 & 0.8 \\
\hline 19 & 98.9 & 98.7 & 98.8 & 1.2 \\
\hline 20 & 98.8 & 98.5 & 98.9 & 1.1 \\
\hline 21 & 98.2 & 98.2 & 98.5 & 1.5 \\
\hline 22 & 98.5 & 98.5 & 98.7 & 1.3 \\
\hline 23 & 98.9 & 98.8 & 98.8 & 1.2 \\
\hline 24 & 98.6 & 98.5 & 98.3 & 1.7 \\
\hline 25 & 98.5 & 98.5 & 98.8 & 1.2 \\
\hline 26 & 98.4 & 98.4 & 99.0 & 1.0 \\
\hline 27 & 98.9 & 98.5 & 99.1 & 0.9 \\
\hline 28 & 98.6 & 98.1 & 98.4 & 1.6 \\
\hline 29 & 98.2 & 98.3 & 98.9 & 1.1 \\
\hline 30 & 98.6 & 97.5 & 98.6 & 1.4 \\
\hline Average & 98.5 & 98.3 & 98.8 & 1.2 \\
\hline
\end{tabular}




\section{Table 4 (on next page)}

Table IV. Model performance based on confusion matrix after 10 -fold cross-validation 
1 Table IV. Model performance based on confusion matrix after 10-fold cross-validation

\begin{tabular}{ccccc}
\hline Dataset & Accuracy (\%) & $\begin{array}{c}\text { Sensitivity } \\
(\%)\end{array}$ & Specificity (\%) & F-Score (\%) \\
\hline Training Dataset & 98.5 & 98.8 & 98.0 & 98.4 \\
Testing Dataset & 98.8 & 99.1 & 98.4 & 98.8 \\
\hline
\end{tabular}




\section{Table 5 (on next page)}

Table V. Performance comparison between different classifiers and the proposed method in breast cancer classification 
Table V. Performance comparison between different classifiers and the proposed method in breast cancer classification.

\begin{tabular}{ccc}
\hline Model & Name of the classifier & Accuracy \\
\hline 1.1 & Fine Tree & $97.20 \%$ \\
1.2 & Medium Tree & $96.90 \%$ \\
1.3 & Coarse Tree & $97.50 \%$ \\
1.4 & Linear SVM & $98.00 \%$ \\
1.5 & Quadratic SVM & $97.70 \%$ \\
1.6 & Cubic SVM & $97.00 \%$ \\
1.7 & Fine Gaussian SVM & $80.70 \%$ \\
1.8 & Medium Gaussian SVM & $97.90 \%$ \\
1.9 & Coarse Gaussian SVM & $98.10 \%$ \\
1.10 & Fine KNN & $79.80 \%$ \\
1.11 & Medium KNN & $97.80 \%$ \\
1.12 & Coarse KNN & $96.30 \%$ \\
1.13 & Cosine KNN & $97.10 \%$ \\
1.14 & Cubic KNN & $97.60 \%$ \\
1.15 & Weighted KNN & $97.80 \%$ \\
1.16 & Boosted Tree & $62.70 \%$ \\
1.17 & Bagged Tree & $97.30 \%$ \\
1.18 & Subspace Discriminate & $95.80 \%$ \\
1.19 & Subspace KNN & $85.40 \%$ \\
1.20 & RUSBoosted Tree & $62.70 \%$ \\
$\mathbf{1 . 2 1}$ & Proposed IANN & $\mathbf{9 8 . 8 0 \%}$ \\
\hline
\end{tabular}




\section{Table 6(on next page)}

Table VI. Comparison of breast cancer classification performance between existing methods in the literature and the proposed method 
Table VI. Comparison of breast cancer classification performance between existing methods in the literature and the proposed method

\begin{tabular}{ccc}
\hline $\begin{array}{c}\text { Author Name and } \\
\text { Reference }\end{array}$ & Method & Accuracy \\
\hline Aalaei et al. [5] & GA- Based ANN & $96.50 \%$ \\
Salama et al. [27] & SMO & $97.70 \%$ \\
Xue et al. [31] & PSO & $92.98 \%$ \\
Nekkaa et al. [32] & MA+SVM & $97.88 \%$ \\
Ouy et al. [36] & APSO-NN & $98.24 \%$ \\
Amrane et al. [48] & KNN & $97.51 \%$ \\
Abdar et al. [49] & SV-NB-3- & $98.07 \%$ \\
The proposed & MetaClassifier & $\mathbf{9 8 . 8 0 \%}$ \\
\hline
\end{tabular}




\section{Table 7 (on next page)}

Table VII. 10-fold cross-validation experimental performance 
Table VII. 10-fold cross-validation experimental performance.

\begin{tabular}{ccc}
\hline $\begin{array}{c}\text { Cross Validation } \\
\text { (10-folds) }\end{array}$ & $\begin{array}{c}\text { Training } \\
\text { Accuracy (\%) }\end{array}$ & $\begin{array}{c}\text { Test } \\
\text { Accuracy }(\%)\end{array}$ \\
\hline K-1 & 99.2 & 97.9 \\
K-2 & 98.6 & 100 \\
K-3 & 99.0 & 100 \\
K-4 & 98.6 & 98.2 \\
K-5 & 97.8 & 98.5 \\
K-6 & 98.2 & 98.2 \\
K-7 & 98.4 & 98.5 \\
K-8 & 98.0 & 98.6 \\
K-9 & 98.8 & 98.8 \\
K-10 & 98.3 & 98.2 \\
Average & 98.5 & 98.7 \\
\hline
\end{tabular}

2 
Figure 1

The basic architecture of an ANN

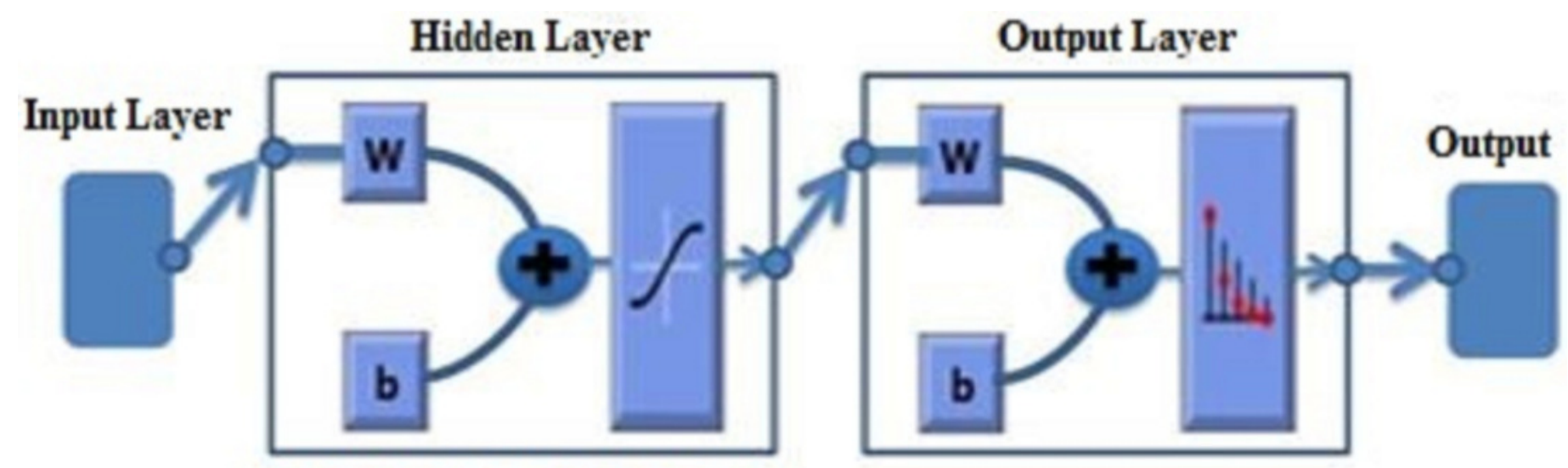


Figure 2

The architecture of a Multi-layer feed-forward neural network with four inputs.

Input Layer

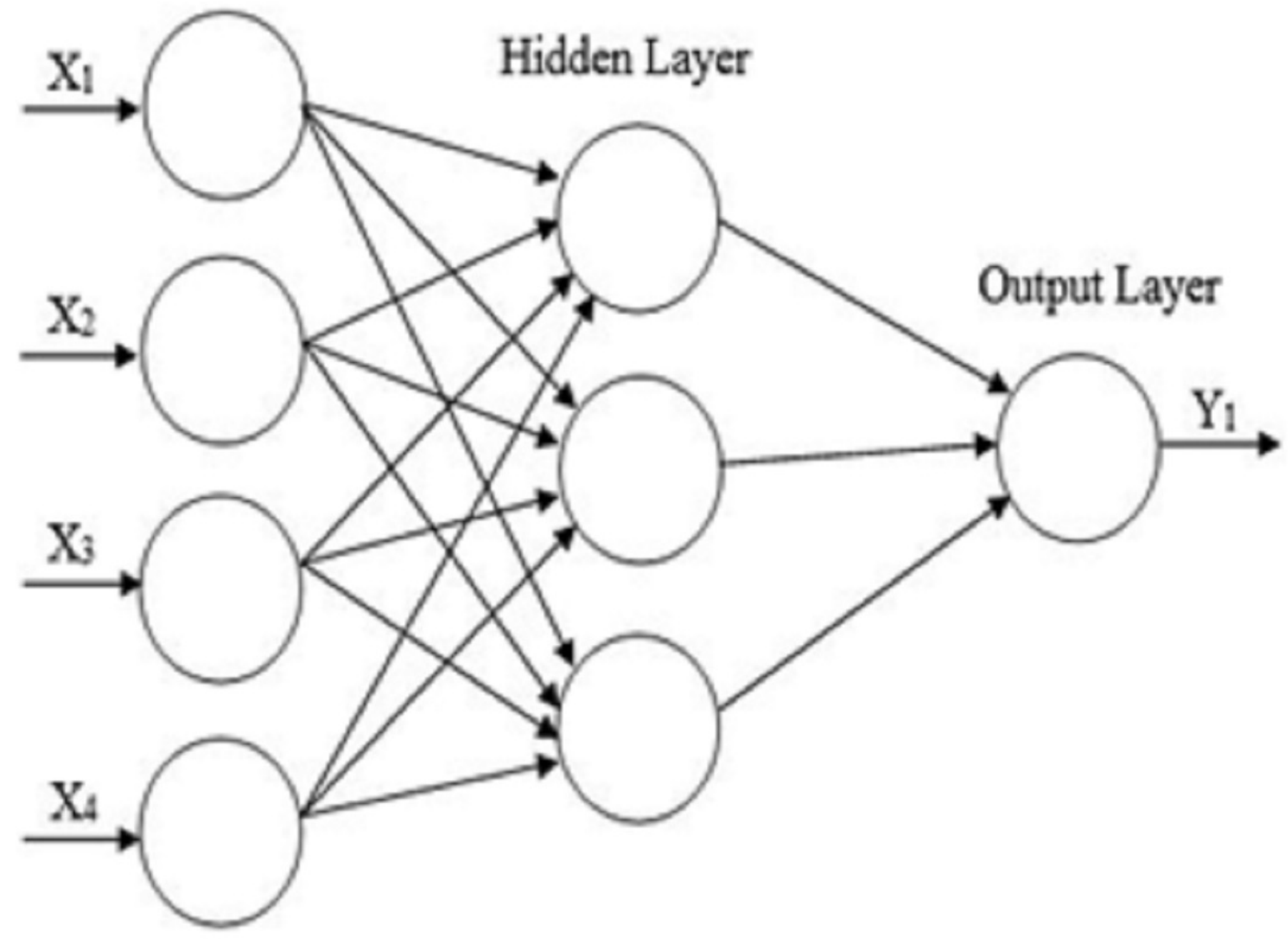


Figure 3

Architecture of the proposed 15 neurons ANN classifier and two output layers

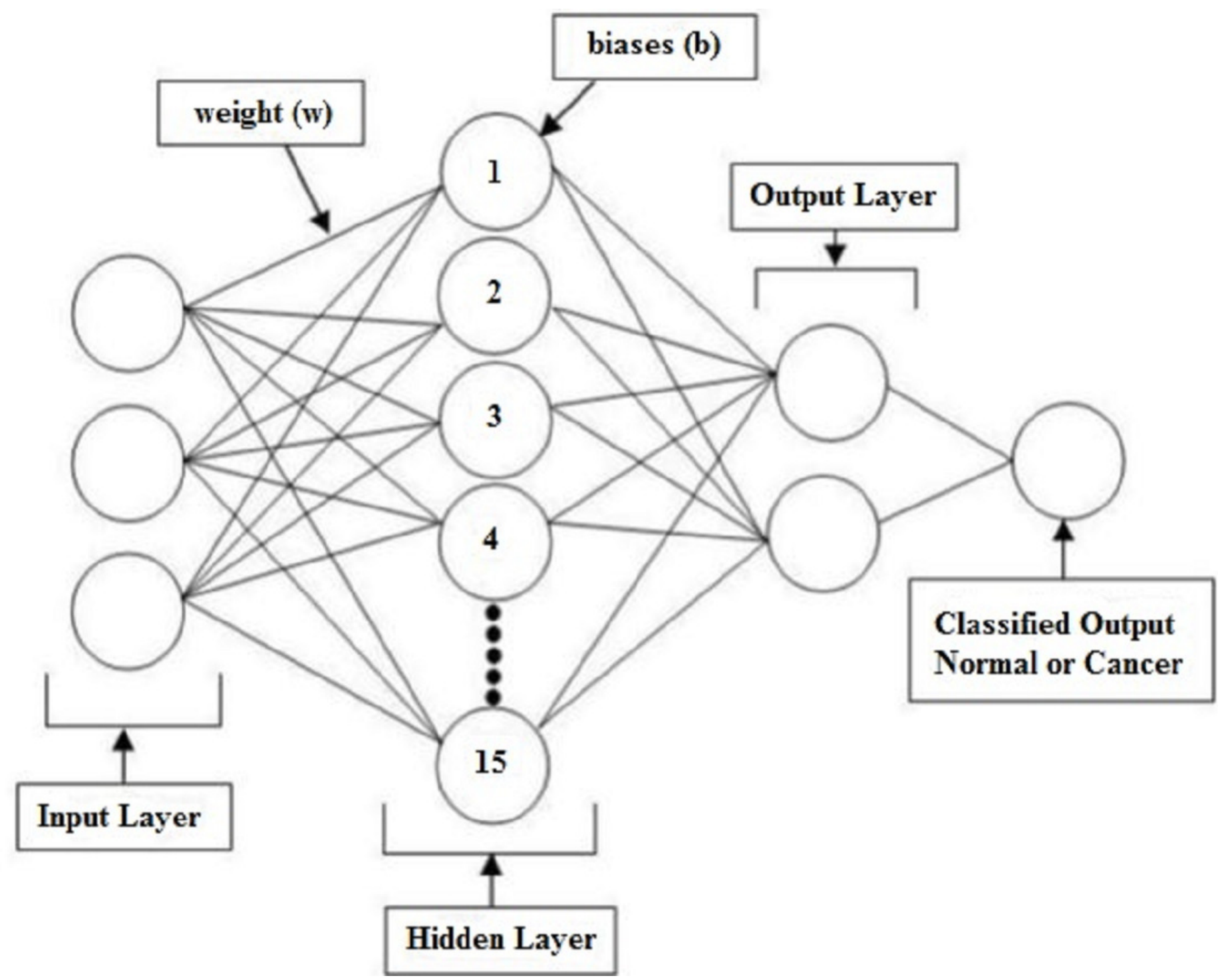




\section{Figure 4}

The proposed Improved-ANN (IANN) cancer classification model

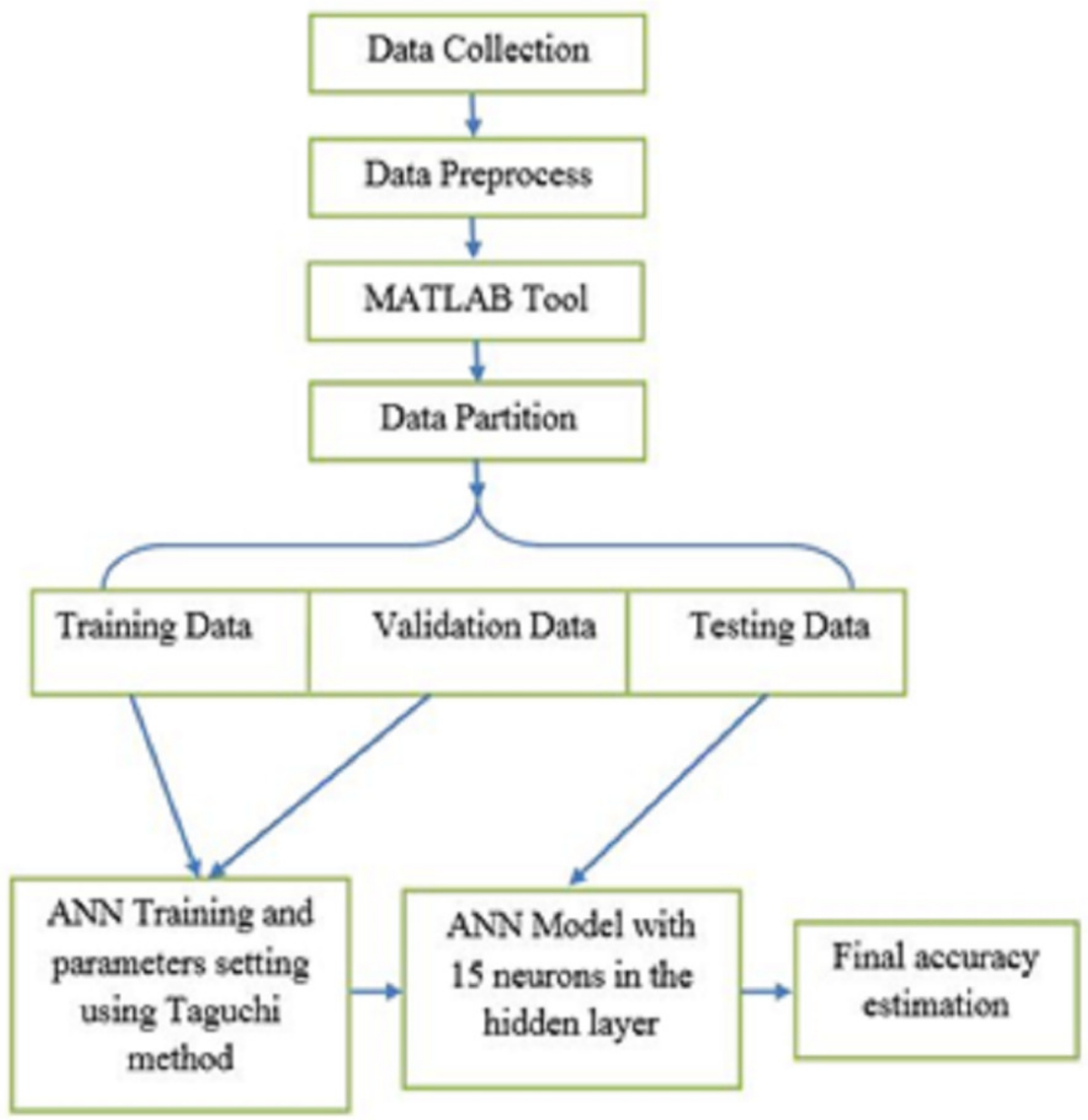


Figure 5

IANN training performance

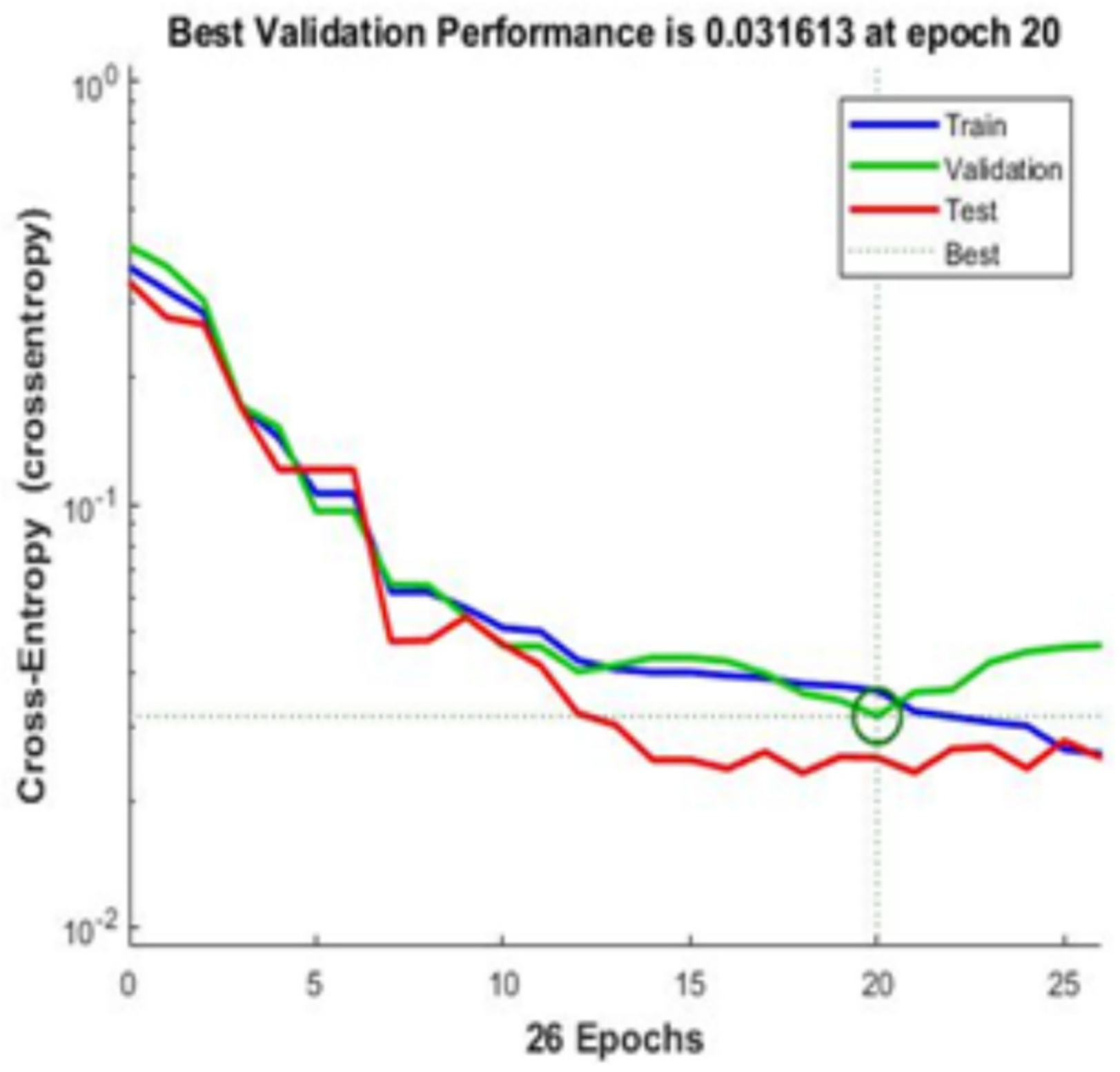


Figure 6

Training state performance
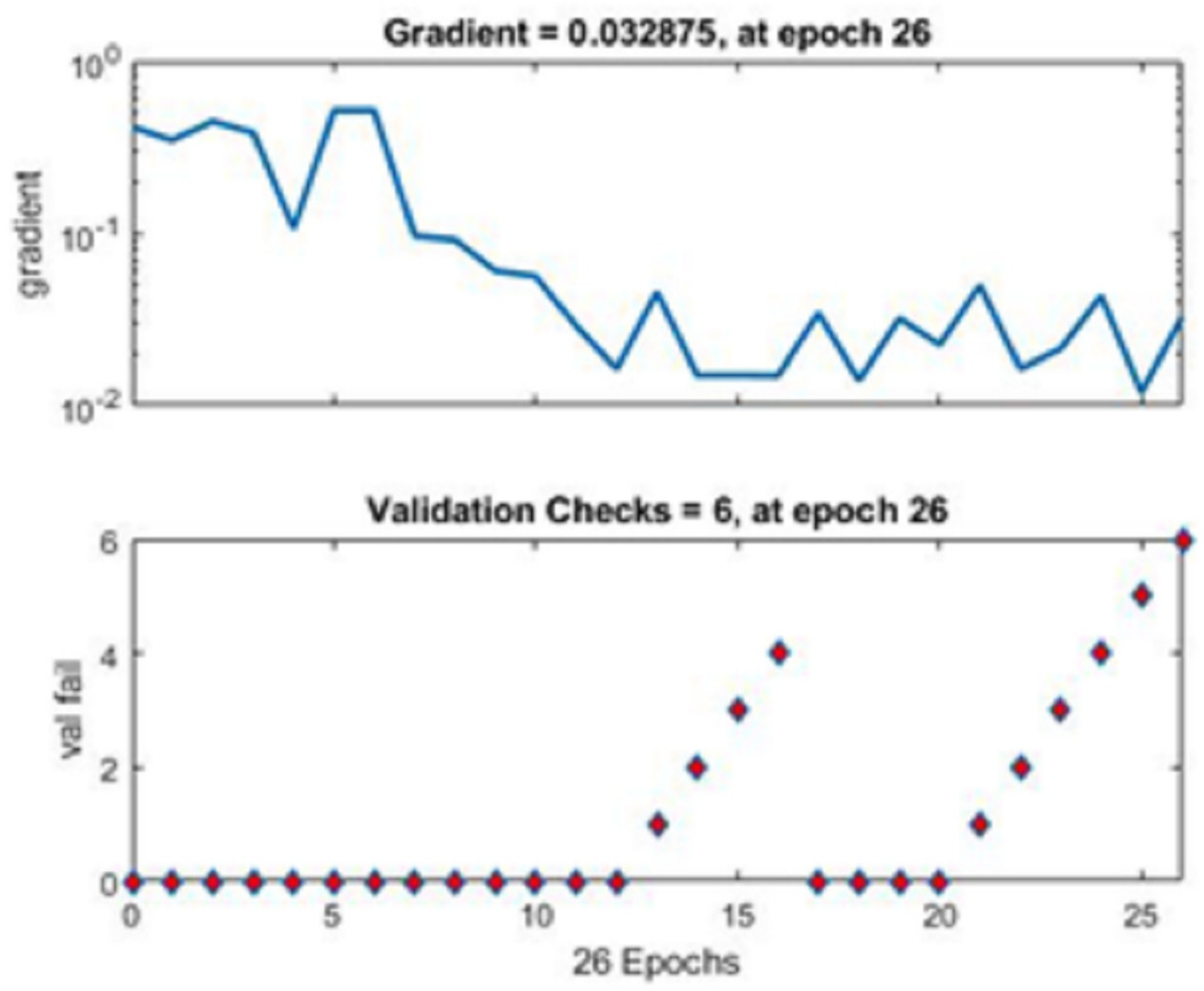


\section{Figure 7}

\section{Error histogram}

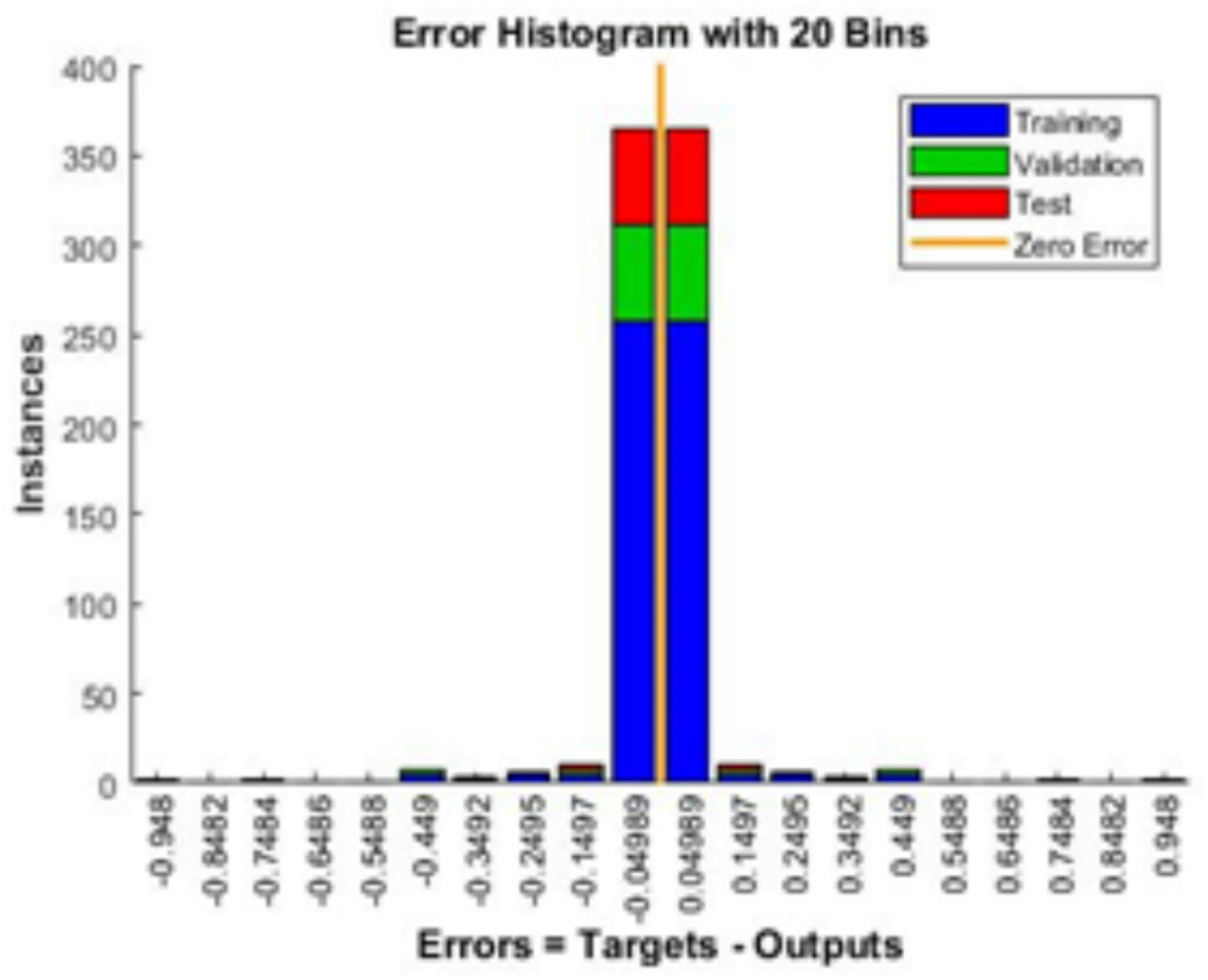




\section{Figure 8}

Performance confusion matrix

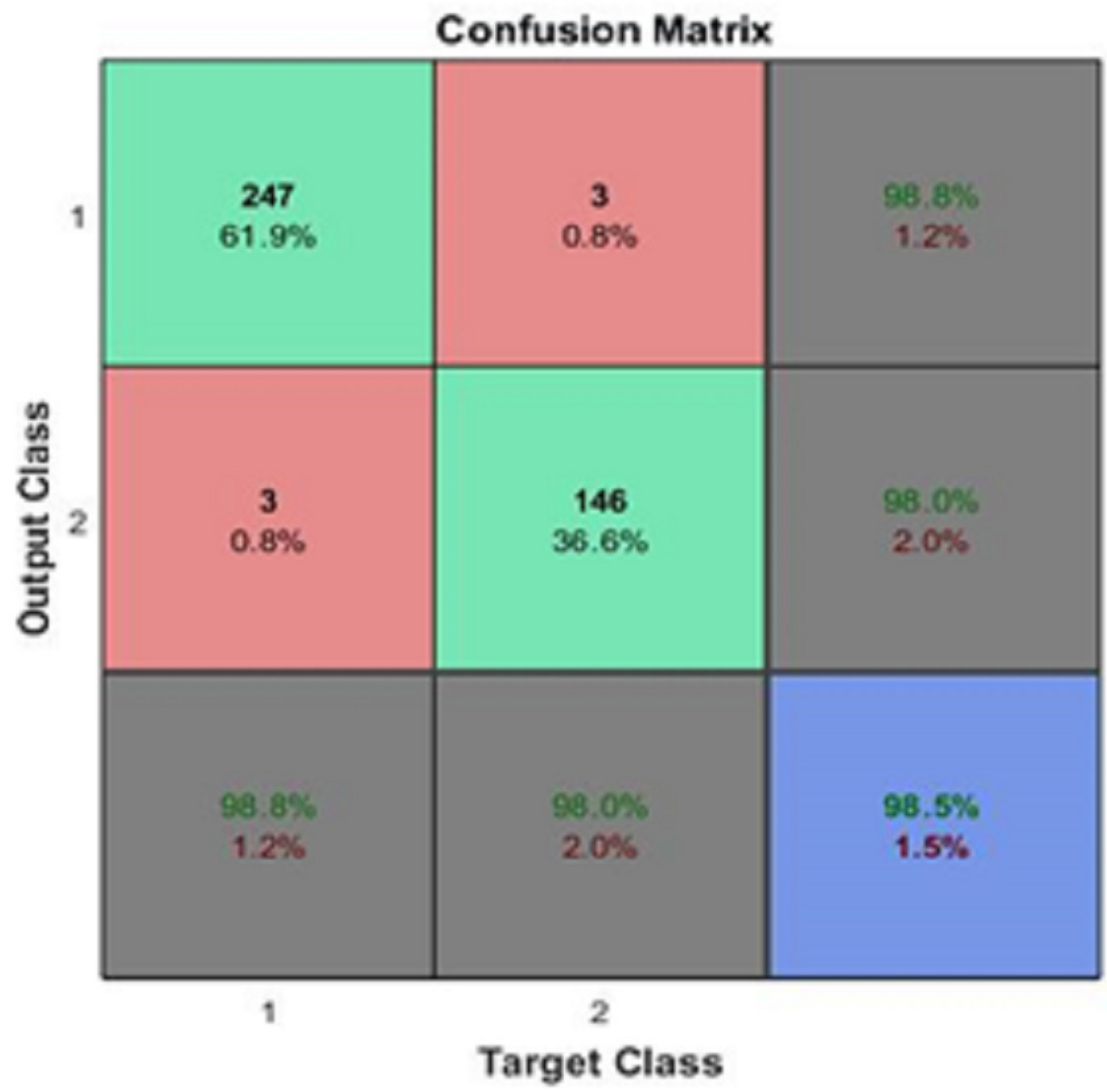


Figure 9

Receiver operating characteristic (ROC) curve

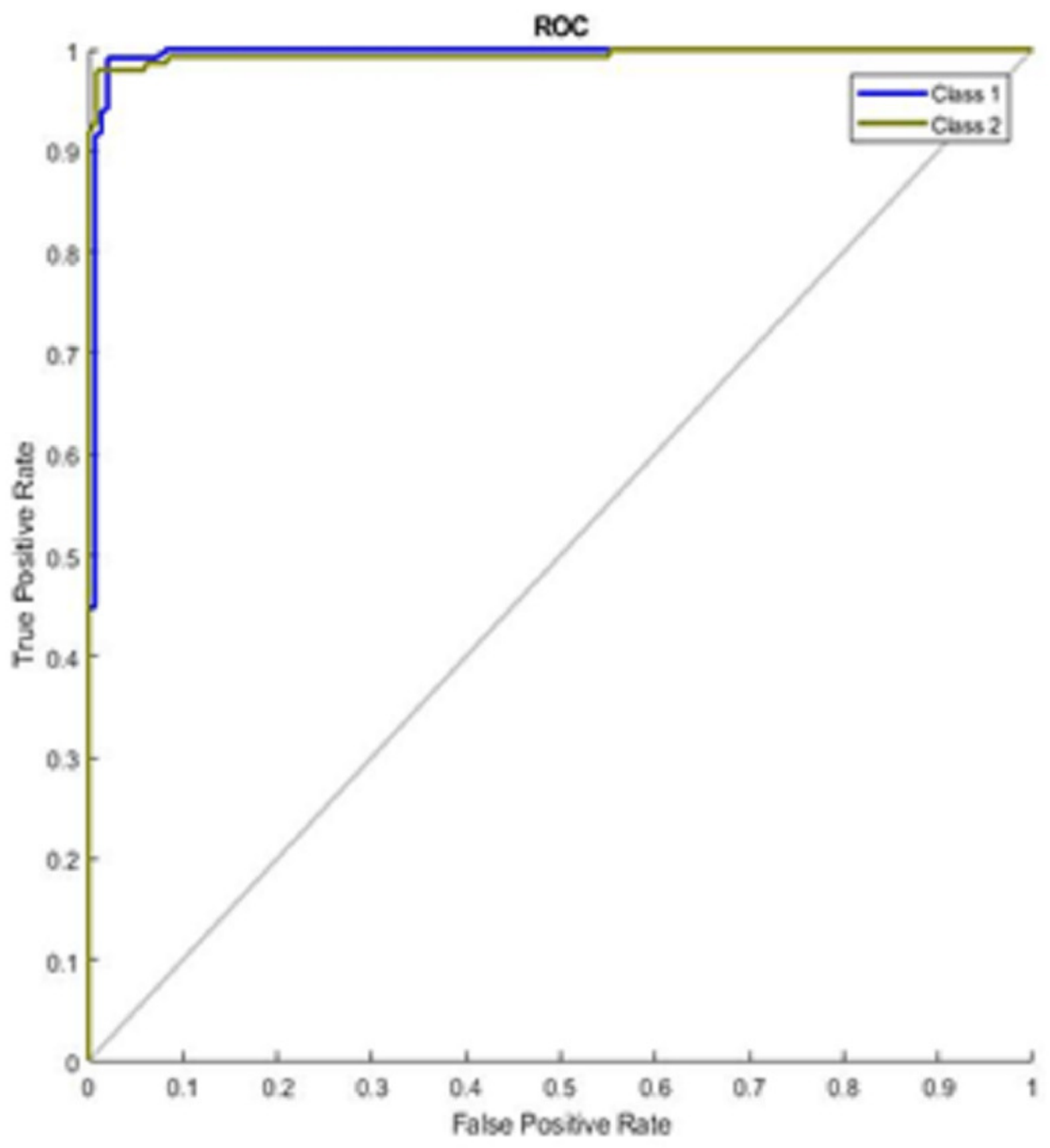




\section{Figure 10}

Test dataset result of breast cancer

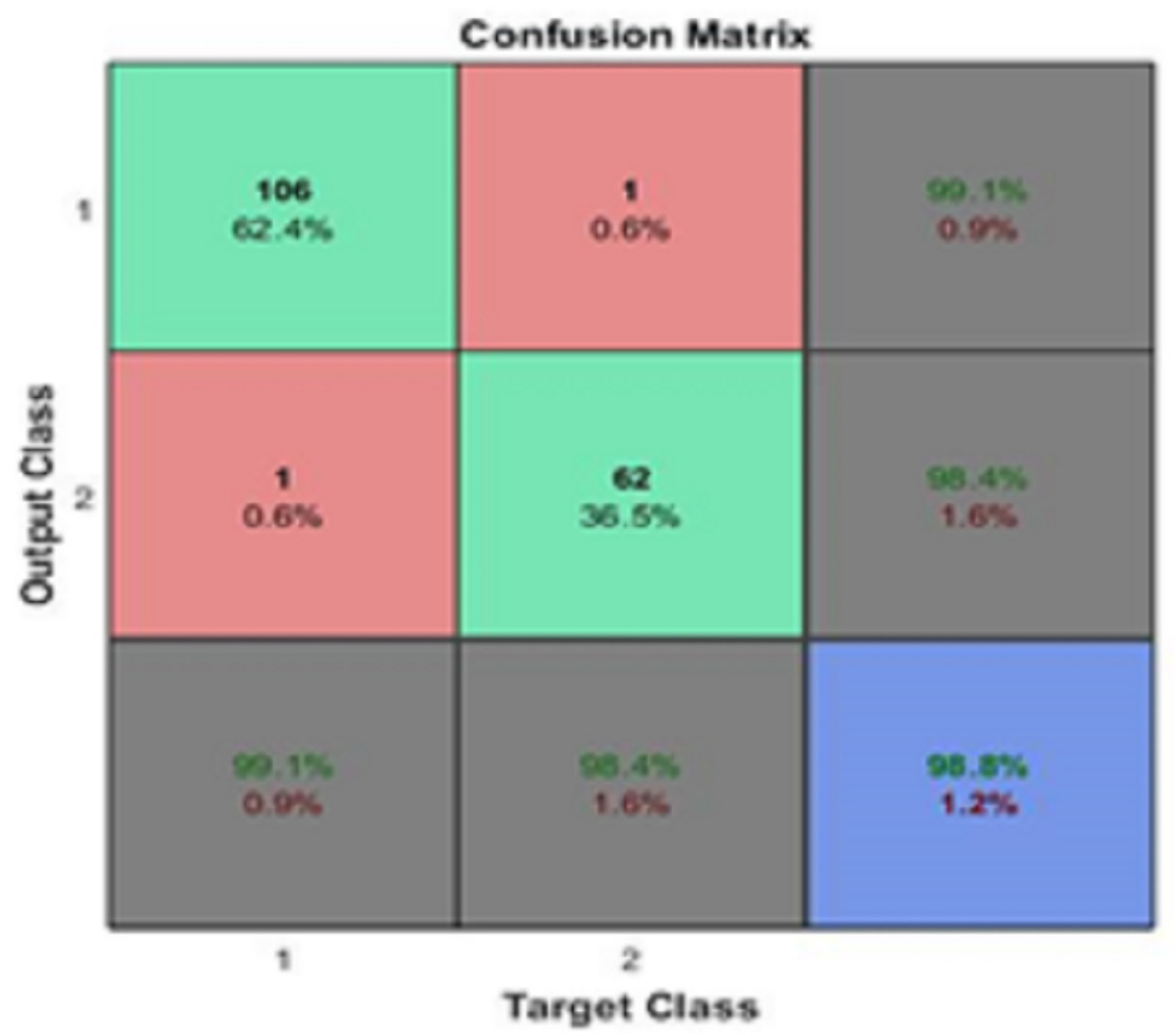


Figure 11

ROC curve with test dataset of breast cancer

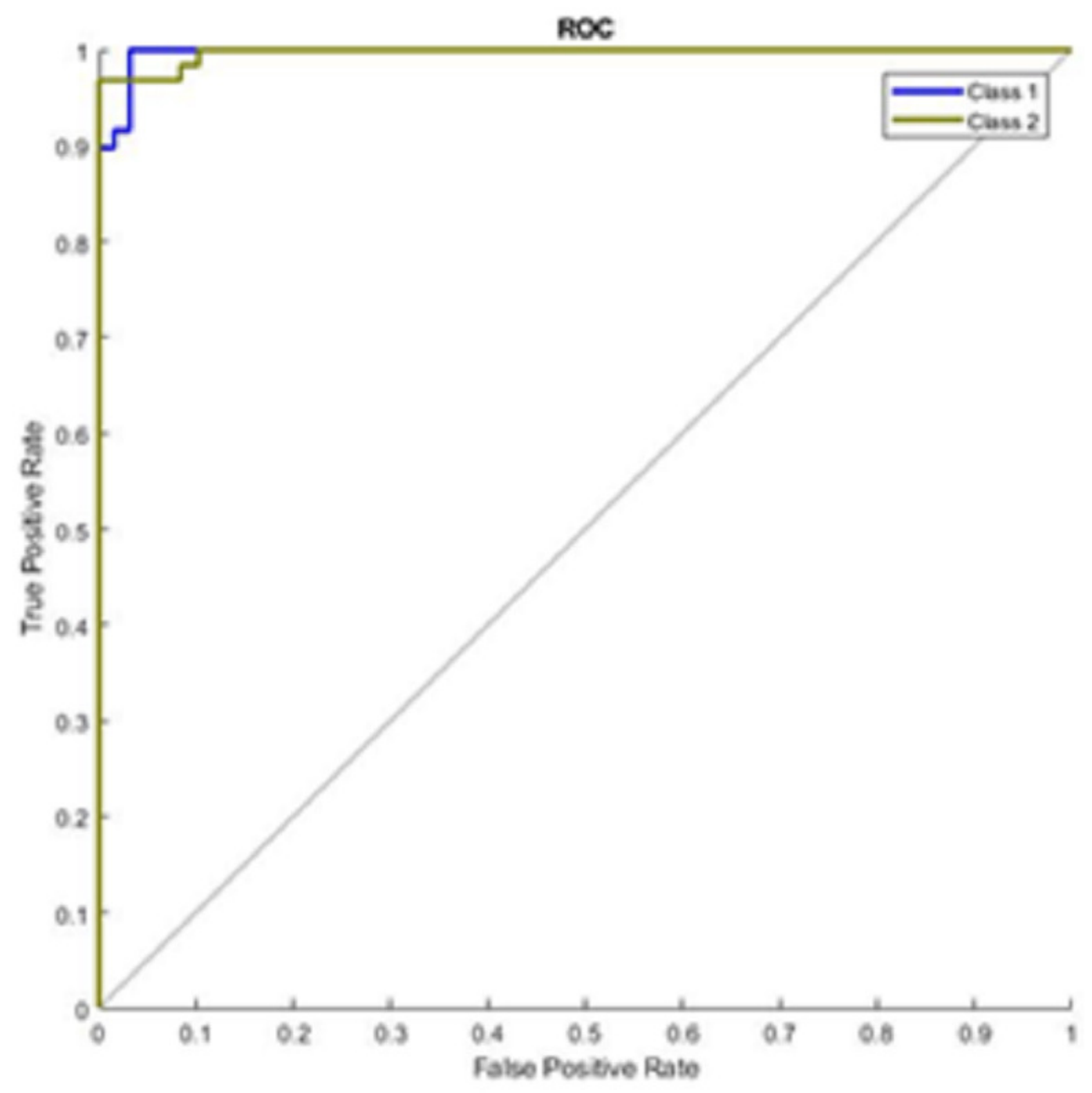

